\title{
Molecular candidates for early-stage flower-to-fruit transition in stenospermocarpic table grape (Vitis vinifera L.) inflorescences ascribed by differential transcriptome and metabolome profiles
}

\author{
Sara Domingos ${ }^{\mathrm{a}, \mathrm{b}}$, Joana Fino ${ }^{\mathrm{c}}$, Octávio S. Paulo ${ }^{\mathrm{c}}$, Cristina M. Oliveira ${ }^{\mathrm{a}}$, Luis F. Goulao ${ }^{\mathrm{b}, *}$ \\ a Linking Landscape, Environment, Agriculture and Food (LEAF), Instituto Superior de Agronomia (ISA), Universidade de Lisboa, Lisbon, Portugal \\ ${ }^{\mathrm{b}}$ BioTrop, Instituto de Investigação Científica Tropical I.P. (IICT), Lisbon, Portugal \\ ${ }^{c}$ Computational Biology and Population Genomics Group, cE3c-Centre for Ecology, Evolution, and Environmental Changes, Faculdade de Ciências, \\ Universidade de Lisboa, Lisbon, Portugal
}

\section{A R T I C L E I N F O}

\section{Article history:}

Received 6 September 2015

Received in revised form 8 December 2015

Accepted 18 December 2015

Available online 6 January 2016

\section{Keywords:}

Fruit set

Grapevine

Metabolic pathways

RNA-Seq

Seedless

\begin{abstract}
A B S T R A C T
Flower-to-fruit transition depends of nutrient availability and regulation at the molecular level by sugar and hormone signalling crosstalk. However, in most species, the identities of fruit initiation regulators and their targets are largely unknown. To ascertain the main pathways involved in stenospermocarpic table grape fruit set, comprehensive transcriptional and metabolomic analyses were conducted specifically targeting the early phase of this developmental stage in 'Thompson Seedless'. The high-throughput analyses performed disclosed the involvement of 496 differentially expressed genes and 28 differently accumulated metabolites in the sampled inflorescences. Our data show broad transcriptome reprogramming of molecule transporters, globally down-regulating gene expression, and suggest that regulation of sugar- and hormone-mediated pathways determines the downstream activation of berry development. The most affected gene was the SWEET14 sugar transporter. Hormone-related transcription changes were observed associated with increased indole-3-acetic acid, stimulation of ethylene and gibberellin metabolisms and cytokinin degradation, and regulation of MADS-box and AP2-like ethylene-responsive transcription factor expression. Secondary metabolism, the most representative biological process at transcriptome level, was predominantly repressed. The results add to the knowledge of molecular events occurring in grapevine inflorescence fruit set and provide a list of candidates, paving the way for genetic manipulation aimed at model research and plant breeding.
\end{abstract}

(C) 2015 Elsevier Ireland Ltd. All rights reserved.

\section{Introduction}

Fruit set, or flower-to-fruit transition, is the stage in which the ovary progresses to a growing young fruit. Fruit cell number and the

\footnotetext{
Abbreviations: ACO,1-aminocyclopropane-1-carboxylate oxidase; AMP, adenosine 5'-monophosphate; bp, base pair; CW, cell wall; cv, cultivar; DEGs, differentially expressed genes; d, days after $100 \%$ cap fall; FDR, false discovery rate; IAA, indole3-acetic acid; IAMT, indole-3-acetate-o-methyltransferase; FPKM, fragments per kilobase of exon per million fragments mapped; FS, fruit set stage; GA, gibberellin; GA20ox, gibberellin 20 oxidase; GO, Gene Ontology; KEGG, Kyoto Encyclopaedia of Genes and Genomes; KOG, euKaryotic Orthologous Groups; PCoA, principal coordinate analysis; PCD, programmed cell death; PMN, Plant Metabolic Network; RNA-Seq, ribonucleic acid sequencing; SAM, S-adenosylmethionine; SNPs, single nucleotide polymorphisms.

* Corresponding author. Present address: Colégio Food, Farming and Forestry, Universidade de Lisboa, Portugal.

E-mail addresses: goulao@reitoria.ulisboa.pt, luisfgoulao@gmail.com (L.F. Goulao).
}

ultimate number of fruits formed are determined during this developmental stage, with decisive impact on the final fruit size and plant yield potential. During the first 2 weeks after full bloom, an abrupt increase in ovary size occurs due to cell multiplication and, to a lesser extent, to cell enlargement [1-4]. During this period, reproductive organs are significantly more sensitive to biotic and abiotic stresses than during later stages of berry development or vegetative growth [5-10], so the success of this developmental stage is critical both to species biological survival and agronomic sustainability.

Nowadays, an increase in table grape world production is clearly evident and, based on consumer preferences, is accompanied by a strong interest in seedless varieties for fresh consumption. Therefore, seedlessness is among the main focus of current table grape breeding programmes [11]. In seeded grapes, flower-to-fruit transition requires pollination and ovary fertilisation toward seed formation. In seedless grapes, two mechanisms can be distinguished: parthenocarpy, which occurs when the ovary is able to develop without ovule fertilisation (e.g., cv Black Corinth), and 
stenospermocarpy, which progresses after pollination and fertilisation but with the embryo aborting 2-4 weeks after fertilisation (e.g., cv Thompson Seedless) [2,12]. In the latter case, partially developed seeds or seed traces can be found.

Natural flower shatter is an event that occurs during fruit set, and generally only $20-30 \%$ of the flowers develop into berries [2]. Fruit set is increasingly sustained by photoassimilates and other nutrients exported from photosynthetically active leaves through the phloem [13] and photosynthesised by the inflorescence itself. Hence, the success of this developmental process depends on factors such as photoassimilate production, transport capacity, and phloem unloading to the fruit. Nonetheless, the main determinant is the fruit sink strength $[14,15]$.

Based on transcript and metabolite analyses during fertilisationdependent and fertilisation-independent fruit formation, it was further verified in the fruit model species tomato (Solanum lycopersicon) that, in addition to nutrient availability, common pathways of flower-to-fruit transition include modulation by sugar and hormone signalling crosstalk and regulation by specific transcription factors from several families [16-24]. However, only few components of the mechanism regulating fruit set have been identified so far. According to the hypothetical model for sugar signalling in seed and fruit set regulation [25], glucose, as the product of sucrose hydrolysis, acts as a signal molecule to repress the expression of programmed cell death (PCD) genes and to promote cell division that, in turn, leads to fruit set. Under severe stress conditions, phloem sucrose import is blocked, which together with a depletion of starch reserves results in reduced glucose levels, triggering the PCD pathway and inhibiting cell division, consequently leading to fruit abortion [26]. Auxins, ethylene, and gibberellins (GAs) are other key players with a prominent role in triggering and coordinating the fruit set developmental process [22]. Auxin signalling is recognised as one of the earliest events in the fruit initiation cascade, particularly the fine-tuning of AUXIN/INDOLE-3-ACETIC ACID, AUXIN RESPONSE FACTOR, and MADS-box gene expression [24]. In addition, fruit set is mediated by activating GA biosynthesis mainly through the up-regulation of GIBBERELLIN OXIDASE 20 (GA20ox) [21]. Polyamine metabolism showed different dynamics according to individual species. Levels of putrescine were shown to decrease in tomato while increasing in plum (Prunus insititia) during fruit set $[24,27]$. Alterations in secondary metabolism were also found during this stage. For instance, ascorbate antioxidant levels declined during the anthesis to post-anthesis transition, while its direct precursor, galactonate-1,4-lactone, increased at the post-anthesis stage [24].

Despite the importance of grapevine, both from an economic point of view and as a genomic model for studying woody fruit species [28,29], to date, the development-related global transcriptomic and metabolomic analyses reported targeted only later stages of berry development [30-35]. In this work, through differential gene expression and metabolite analyses, we aimed to identify the putative molecular cues responsible for initial flowerto-fruit transition in stenospermocarpic table grapes, which have a profound impact on the later stages of berry development.

\section{Methods}

\subsection{Sample collection}

Grape inflorescences were collected from 7-year-old cv Thompson Seedless commercial vines (Vitis vinifera $\mathrm{L}$.) that were grafted on a 140 Ruggeri rootstock and spaced $3 \times 3 \mathrm{~m}$. The commercial vineyard is located in Ferreira do Alentejo, southern Portugal and was managed following standard fertilisation, irrigation, and pest-management practices. Vines were grown under an overhead trellis system covered with plastic, providing favourable conditions for a compressed and homogeneous flowering period. Climate conditions (photosynthetically active radiation, temperature, and relative humidity) were monitored above the vine canopy (WatchDog MicroStation, Spectrum Technologies, Aurora, USA) and shown to be maintained during the sampling period (Supplementary Fig. A.1). To guarantee that the 2-3-day period required after pollination for fertilisation to be completed [9] was fully targeted, the inflorescence sampling was conducted at three time points (Fig. 1): fruit set stage 1 (FS1; 3 days after $100 \%$ cap fall ( 3 d)), FS2 ( 5 d), and FS3 (7 d). One hundred per cent cap fall corresponded to stage 69 of the $\mathrm{BBCH}$ scale [36]. At each time point, three inflorescences were selected from a five-vine plot based on uniformity of blossom. An inflorescence was considered to be homogenous when more than $90 \%$ of the flowers showed to be at the same stage. Each biological replicate was made up of an inflorescence, deprived from the rachis, immediately frozen in liquid nitrogen and subsequently powdered and stored at $-80^{\circ} \mathrm{C}$ until use.

\subsection{RNA whole transcriptome deep sequencing}

Total RNA was extracted and purified from $100 \mathrm{mg}$ frozen material independently for each biological sample using the RNeasy Plant RNA Extraction Kit and RNase-Free DNase Set (Qiagen, Hilden, Germany) following the manufacturer's instructions, modified by replacing the kit's extraction buffer by $100 \mathrm{mM}$ Tris- $\mathrm{HCl}, 2 \%(\mathrm{w} / \mathrm{v})$ CTAB, $25 \mathrm{mM}$ EDTA, and $2 \mathrm{M}$ $\mathrm{NaCl}$ buffer [37]. When traces of contaminant genomic DNA were detected after standard PCR amplification of the Actin 1 (ACT1) gene (GenBank Accession: XM_002282480.3; forward primer: 5'-CTTCCAGCCATCTCTCATTGG-3' and reverse primer: 5'- TGTTGCCATAGAGGTCCTTCC-3'), RNA samples were further digested with RNase-free DNase I (Ambion, Life Technologies, Carlsbad, USA). RNA integrity and purity were evaluated by visual inspection of ribosomal bands after 1.5\% agarose gel electrophoresis and through a 2100 Bioanalyzer system (Agilent Technologies, Santa Clara, USA) readings. Poly(A) mRNA isolation, cDNA synthesis, library generation, indexing, cluster generation, and RNA-Seq analyses by Illumina HiSeq 2000 RNA sequencing of 100-bp pairedend reads were carried out by LGC Genomics (Berlin, Germany), using commercial services.

\subsection{Alignment and analysis of Illumina reads}

The raw Illumina 100-bp paired-end sequences were deposited in the NCBI Sequence Read Archive under accession numbers SRS:853491, SRS:879704, SRS:879828, SRS:879847, SRS:879856, SRS:882229, SRS:882241, SRS:882246, and SRS:882247.

Poor quality base reads and adapter sequences were trimmed using Trimmomatic version 0.32 software [38]. Trimmed reads were mapped back to the reference genome [28] for quantification of gene expression levels and global transcript expression profiling within each time point using Tophat2 version 2.0.12 [39] with the parameters -D 15 -R 2 -L 22 -i S,1,1.15 as well as end-to-end mode. The numbers of reads mapped to each gene per sample were extracted from the output bam files of Tophat2 using the in-house python script bamzinga (https://github.com/Nymeria8/bamzinga). Quantification and normalisation of gene expression values by fragments per kilobase of exon per million fragments mapped (FPKM) was calculated by the Cufflinks package cuffdiff version 2.2.1 [40]. The Mfuzz R package version 2.26.0 [41] processed the expression data to generate clustering plots with missing data and low standard deviation filtering, with number of centres set to 9 .

The RNA-Seq data were also used to compare the cv Thompson Seedless sequence with the seeded variety Pinot Noir reference genome [28]. Across the expressed genes in 'Thompson' inflo- 


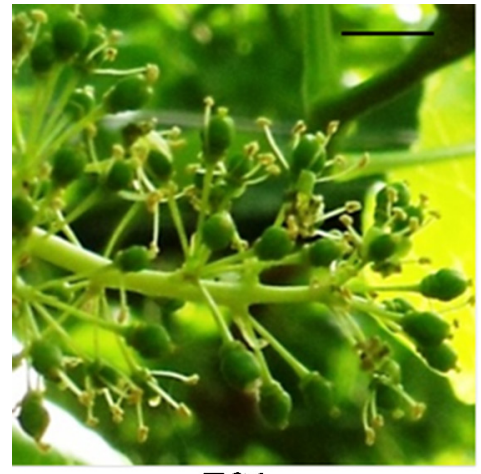

FS1

(3d)

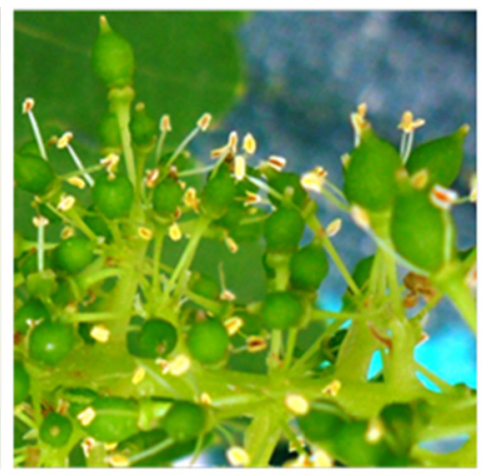

FS2

(5d)

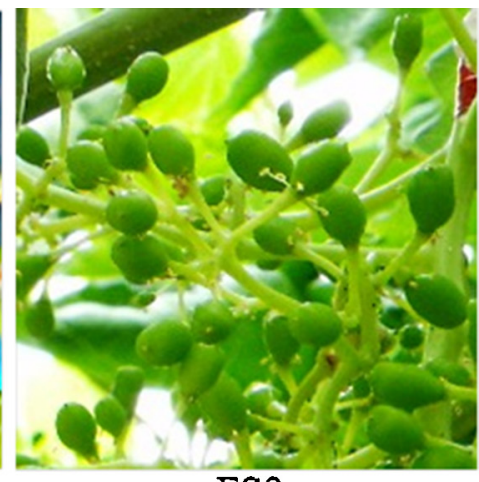

FS3

(7d)

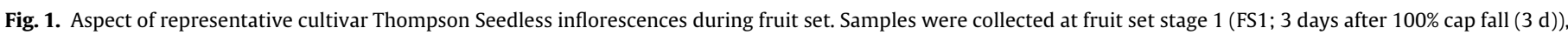
FS2 (5 d), and FS3 (7 d). Scale bar is $0.6 \mathrm{~cm}$.

rescences under the conditions of the assay, single nucleotide polymorphisms (SNPs) and post-transcriptional processing events, such as constitutive and alternative splicing, were searched. For the detection of SNPs, Atlas SNP software was used [42]. The Cufflinks program cuffmerge was used to identify new splicing events between the reference genome and our dataset. To increase accuracy, we conservatively required that at least 10 independent reads mapped in the isoform and at least 10 reads mapped across the entire exon-exon junction.

\subsection{Differential gene expression, functional annotation, and pathway analysis}

Statistical analysis to identify differentially expressed genes (DEGs) between time points was conducted using the R package version 3.0.2 from Bioconductor, DESeq2 version 1.4.5 [43], considering an estimation of size factors, a false discovery rate (FDR) of 0.05 , and $\mathrm{a}-1.5 \geq \log _{2}$ fold change $\geq 1.5$.

To obtain the euKaryotic Orthologous Groups (KOG) annotation of grapevine genes, Rapsearch2 [44] was used to search against functional proteins from Arabidopsis thaliana in the database [45], considering an e-value cutoff of $10^{-5}$. From the total DEGs identified, those that were not automatically assigned to a specific KOG functional category were either ascribed in the KOG categories according to the gene description or classified as 'other' or 'of unknown function'. For additional gene assignment in the Kyoto Encyclopaedia of Genes and Genomes (KEGG [46]) and Gene Ontology (GO) annotation, Rapsearch2 similarity searches were locally conducted against a non-redundant ('nr') peptide database. The output was submitted to an in-housedeveloped script, Rapsearch2XML (https://github.com/Nymeria8/ Rapsearch2Xml) and then to Blast2GO [47] for enzyme identification, metabolic pathway assignment, and functional annotation using GO terms. GO enriched categories were assigned using the R Bioconductor package topGO version 2.18.0 [48], using a Fisher's exact test and FDR correction of 0.01 .

Similarity of expression profiles between biological replicates was determined by Pearson correlation coefficient analyses with R 3.1.2 software using natural logarithm (ln)-transformed read counts for the DEGs as input. All the analyses considered three independent biological replicates per treatment sequenced, providing higher statistical robustness and data validation.

\subsection{Global metabolomic analysis}

About $200 \mathrm{mg}$ of powdered material from each of the three biological replicates collected at each time point were lyophilised, extracted with methanol, and analysed using the integrated platform developed by Metabolon ${ }^{\circledR}$ (Durham, USA), consisting of a combination of three independent approaches: (1) ultrahigh performance liquid chromatography/tandem mass spectrometry (UHLC/MS/MS2) optimised for basic species, (2) UHLC/MS/MS2 optimised for acidic species, and (3) gas chromatography/mass spectrometry (GC/MS). Methods were performed as previously described [49-51].

\subsection{Differentially quantified metabolites and mapping onto metabolic pathways}

Raw area counts for each biochemical compound were rescaled by dividing each sample's value by the median value for the specific biochemical. Statistical analysis of the data was performed using Welch's two-sample $t$-tests with Array Studio (Omicsoft) software. Mapping of named metabolites was performed onto general biochemical pathways, as provided in the KEGG databases and Plant Metabolic Network. Box plots were generated for those compounds that showed a significant change using both the Welch two-sample $t$-test $(p$-value $\leq 0.05)$ and $\mid \log _{2}$ fold change $\mid \geq 1$.

\subsection{Global analysis of transcriptomic and metabolomic profiling}

Data for transcript and metabolite profiling analyses was lntransformed, and normal distribution was verified by histogram plotting of the number of reads and metabolites per sample using the $\mathrm{R}$ software package. For exploratory data analyses, principal coordinate analysis ( $\mathrm{PCOA}$ ) was conducted based on the pairwise correlation matrix using the NTsys-PC version 2.20e software package [52]. The DCENTER module was used to transform the symmetric matrix to scalar product and EIGEN for eigenvalue decomposition to identify orthogonal components of the original matrix modules. A minimum-spanning tree was calculated and superimposed to facilitate the visualisation of the distances between operational units. R statistical software was used for heat map construction. Heat map-associated hierarchical clustering and approximate unbiased and bootstrap probability $p$-values were calculated using pvclust version 1.3.2 [53] with the UPGMA method built from the correlation distance measures and 1000 bootstrap replications. 
Table 1

RNA-Seq data overview. Numbers of 100 -bp reads obtained at each fruit set stage sequenced, before and after data trimming (means of three independent biological replicates \pm standar derror (SE)).

\begin{tabular}{llll}
\hline Fruit set stage & $\begin{array}{l}\text { Raw read pairs } \\
(\mathrm{x} 1000)\end{array}$ & $\begin{array}{l}\text { Read pairs after } \\
\text { trimming } \\
(\mathrm{x} 1000)\end{array}$ & $\begin{array}{l}\text { \% of remaining } \\
\text { reads }\end{array}$ \\
\hline FS1 & $28494 \pm 1209$ & $26517 \pm 1192$ & $93.0 \pm 0.4$ \\
FS2 & $36342 \pm 5193$ & $33045 \pm 4534$ & $91.1 \pm 1.5$ \\
FS3 & $24725 \pm 603$ & $22765 \pm 462$ & $92.1 \pm 0.5$ \\
\hline
\end{tabular}

\section{Results}

\subsection{Transcriptome analysis by RNA-Seq}

Nine RNA-Seq 100-bp paired-end read libraries were prepared from poly(A) RNA extracted from grape inflorescences, corresponding to the three time points sampled (FS1, FS2, and FS3), each represented by the three independent biological replicates collected. Each cDNA library resulted in $\approx 29$ million 100 -bp pairedend reads. An overview of the raw read data is given in Table 1 . The results showed that $8 \%$ of the reads were removed since they overpass the threshold cutoff after being trimmed based on the presence of Illumina adapters or low quality bases. The majority $(62 \%)$ of the total number of reads could be mapped back to the reference genome. An additional $1.7 \%$ of reads were mapped to multiple locations within the reference genome sequence, and these reads were discarded. Statistics of each sample mapping are provided in detail in Table 2 and Supplementary Fig. A.2.

Transcription of 25703 grapevine genes was detected in at least one time transition, based on cuffdiff average FPKM across replicates. The class of 10-200 FPKM values included a higher number of genes in the three time points sampled (Table 3 ).

\subsection{Genome functional annotation}

Functional annotation of the genome was required for GO enrichment analyses and gene categorisation (see sub-sections 3.5 and 3.7). The overall functional annotation and gene assignment was made using sequence similarity searches by Rapsearch2 against the KOG and NCBI 'nr' databases. The Vitis genome is $96.9 \%$ annotated, which corresponds to 29048 of the total 29971 identified genes in the grapevine genome. KOG annotation assignments classified 17385 genes (58.0\% of the genome) distributed among 27 different categories, of which the most significant and representative were signal transduction mechanisms (7.5\%), post-translational modification, protein turnover, and chaperones (5.0\%), and transcription (3.1\%). Based on the KEGG annotation of the genome, 2281 genes were identified and assigned to at least one of the 130 pathways. Predominant pathways were purine metabolism, starch and sucrose metabolism, and thiamine metabolism, with 433, 274, and 226 assigned unigenes, respectively. Using GO terms, 14915 genes were categorised, annotated, and distributed into three main GO domains (Supplementary Fig. A.3).
Table 3

Classes of transcript abundance at each fruit set (FS) stage. Numbers of transcripts detected at various levels of abundance at each time point, as calculated by fragments per kilobase of exon per million fragments mapped (FPKM), considering the data from three biological replicates.

\begin{tabular}{llll}
\hline & FS1 & FS2 & FS3 \\
\hline FPKM $>$ 400 & 341 & 355 & 321 \\
FPKM 200-400 & 484 & 473 & 471 \\
FPKM 10-200 & 11818 & 11869 & 11875 \\
FPKM 1-10 & 7098 & 6941 & 7082 \\
FPKM $<1$ & 4944 & 5240 & 4765 \\
Total & 24685 & 24878 & 24484 \\
\hline
\end{tabular}

The 'Thompson Seedless' transcriptome was compared to the reference genome for polymorphisms. From the total 104595 SNPs identified, 71258 were found to be located in coding regions, 22 in introns, 14128 in exons, and 33315 in uncharacterised regions. The analysis of the transcribed portion of the 'Thompson Seedless' genome also allowed for identifying 497 new splice junctions (Supplementary Table B.1).

\subsection{Distribution of gene expression patterns}

The 25703 detected expressing genes were grouped into nine pre-defined clusters, bringing together genes with similar expression patterns during the three time points sampled (Fig. 2). The cluster represented by the highest number of genes was Cluster 9 , composed of a total of 3699 members. On the opposite end, Cluster 3 was represented by 2044 genes. Selection of KOG annotations per cluster retrieved 24 categories associated with the genes represented. With the exception of Cluster 4 , in which post-translational modification, protein turnover, and chaperones comprised the most represented category, all clusters were enriched in signal transduction mechanism-related genes. Cluster 4 was also differentiated by a higher representation of genes associated with translation, ribosomal structure and biogenesis.

\subsection{Overall transcriptome profile}

A total of 496 DEGs during at least one of the time transitions investigated was identified. The list of all genes significantly affected during fruit set, its annotation regarding functional categories and gene code identification, the pattern-related gene cluster and the respective fold change are given in Supplementary Table B.2. The expression pattern represented by Cluster 1 showed the highest number of DEGs, with 181 genes. During the 4-day period defined, the highest difference in gene expression occurred during the transition from FS1 to FS2, with 307 DEGs observed, while in the following 2 days, from FS2 to FS3, only 137 genes showed differential expression. Considering the whole timespan investigated, 108 additional genes showed statistical significance in expression from FS1 to FS3 (Fig. 3 and Supplementary Table B.2).

PCoA and hierarchical clustering based on the 496 DEGs are shown in Fig. 4. Principal coordinate (PC) 1 and PC2 in combination explained $71.04 \%$ of the total variance of the dataset (Fig. $4 \mathrm{~A}$ ). In general, PC1 differentiated FS1 from the remaining clusters, while

Table 2

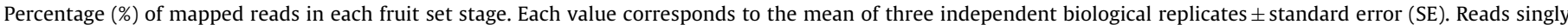
mapped are those that aligned concordantly, while their mate pair was not mapped by the software.

\begin{tabular}{|c|c|c|c|c|c|}
\hline Fruit set stage & Mapped reads & $\begin{array}{l}\text { Paired reads } \\
\text { aligned } \\
\text { concordantly }\end{array}$ & $\begin{array}{l}\text { Multiple } \\
\text { alignments of } \\
\text { read pairs }\end{array}$ & $\begin{array}{l}\text { Reads singly } \\
\text { mapped }\end{array}$ & $\begin{array}{l}\text { Multiple } \\
\text { alignments of } \\
\text { single reads }\end{array}$ \\
\hline FS1 & $61.5 \pm 4.8$ & $54.6 \pm 4.4$ & $1.63 \pm 0.03$ & $6.90 \pm 0.44$ & $1.70 \pm 0.06$ \\
\hline FS2 & $66.0 \pm 3.1$ & $58.7 \pm 2.8$ & $1.63 \pm 0.03$ & $7.30 \pm 0.37$ & $1.73 \pm 0.03$ \\
\hline FS3 & $59.8 \pm 3.4$ & $53.0 \pm 3.1$ & $1.63 \pm 0.03$ & $6.77 \pm 0.35$ & $1.73 \pm 0.03$ \\
\hline
\end{tabular}


Cluster 1

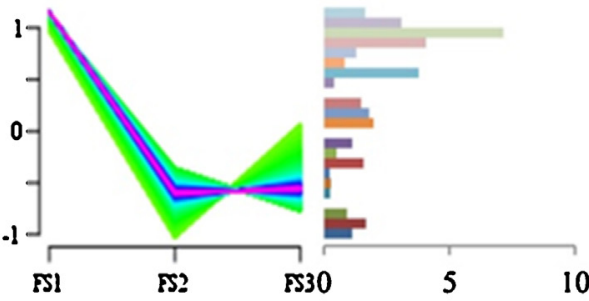

Cluster 4
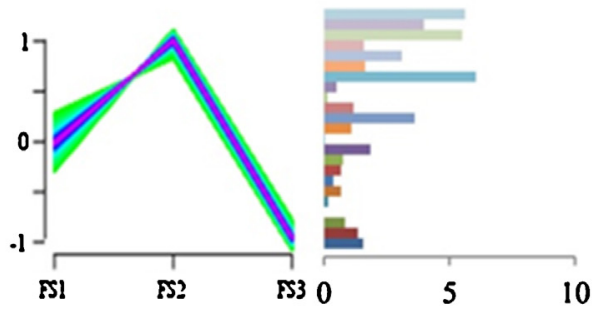

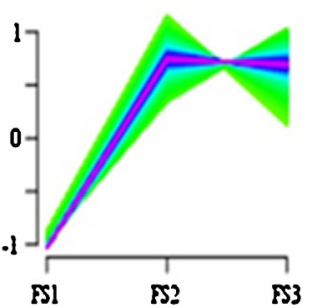

Cluster 3
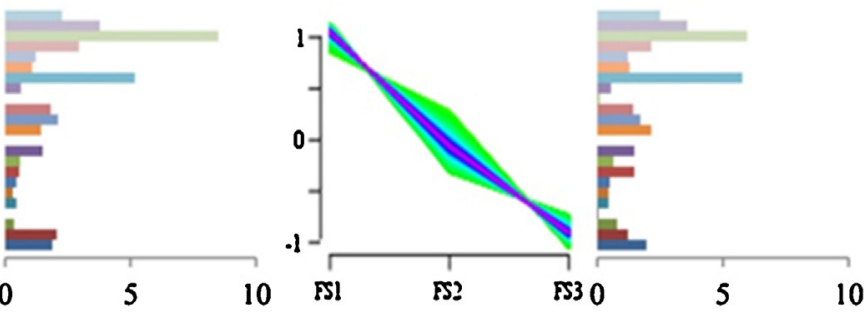

Cluster 7

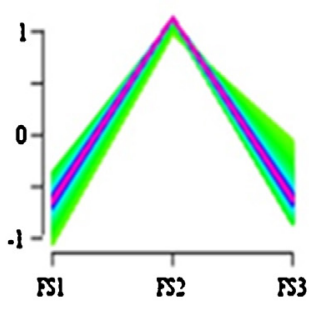

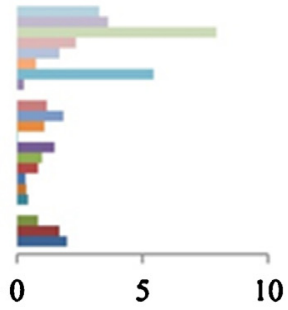

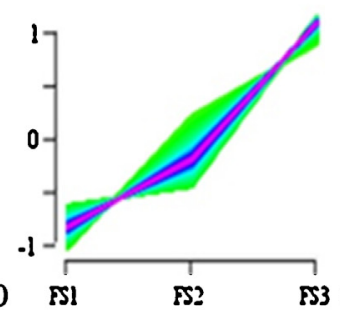

Cluster 5
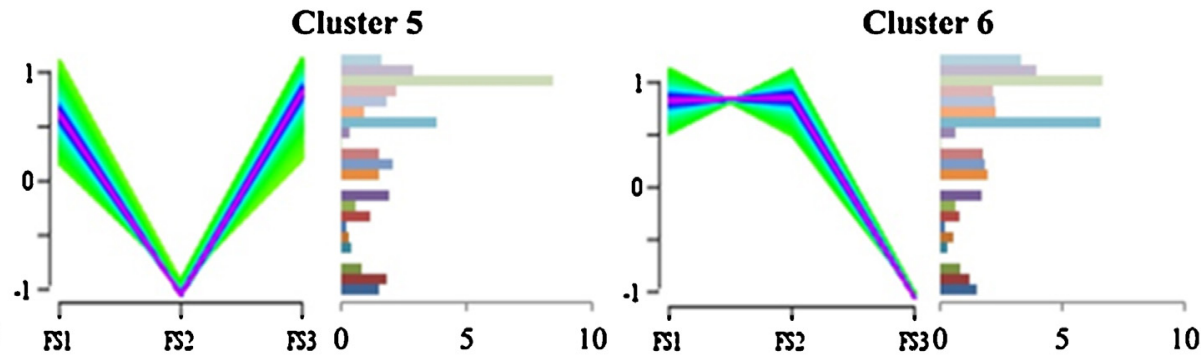

Cluster 6

= Translation, ribosomal structure and biogenesis
= Signal transduction mechanisms
= RNA processing and modification
= Posttranslational modification, protein tumover, chaperones
= Nuclear structure
= Intracellular trafficking, secretion, and vesicular transport
= Extracellular structures
- Defense mechanisms
- Coenzyme transport and metabolism
- Cell wall/membrane/envelope biogenesis
- Cell cycle control, cell division, chromosome partitioning
- Amino acid transport and metabolism

Cluster 8
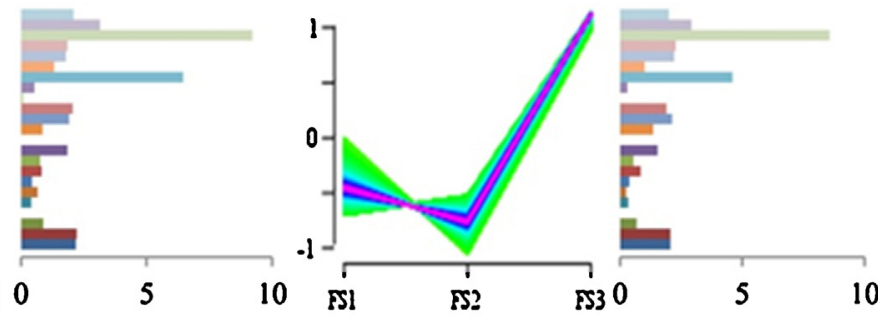

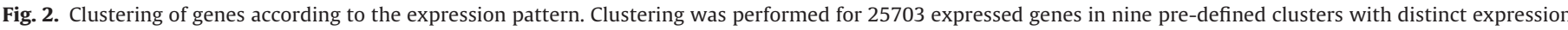

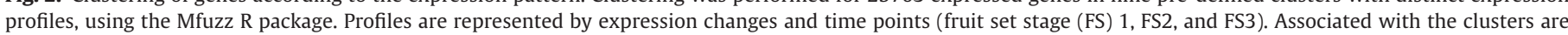

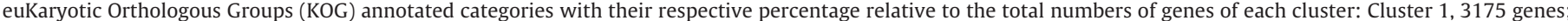
Cluster 2, 2860 genes; Cluster 3, 2044 genes; Cluster 4, 2382 genes; Cluster 5, 2915 genes; Cluster 6, 2409 genes; Cluster 7, 3199 genes; Cluster 8, 3019; Cluster 9, 3699.

PC2 allowed for distinguishing FS2 from FS3 samples. In each case, biological samples were clustered together, and they are directly connected by the minimum-spanning tree. These results indicate that inflorescences from the sampled time points were affected significantly in the overall transcriptome dynamics, providing a suitable experimental dataset. Hierarchical clustering (Fig. 4B) showed the association among samples according to the overall transcriptome profile. Biological replicates collected at each stage were clustered together with strong confidence based on bootstrap analyses and Pearson correlations (Fig. 4B and Supplementary Fig. A.4).

\subsection{GO enrichment analysis}

Enriched categories of DEGs are unevenly represented across the distribution of categories of the grapevine genome, suggesting that predominance of a given pathway is not observed by chance. From a total of 496 DEGs enriched in 188 GO terms, a high proportion (101) of GO terms corresponded to biological processes-81 


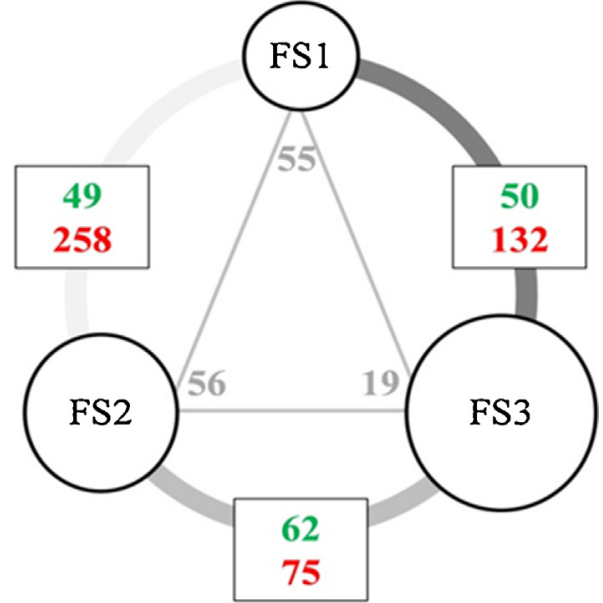

Fig. 3. Diagram showing the numbers and trends of differentially expressed genes (DEGs) between each of the three time points investigated during fruit set. Values indicate genes passing cutoff values of $-1.5 \geq \log _{2}$ fold change $\geq 1.5$ and false discovery rate $(F D R)=0.05$. Green and red values indicate up-regulated and downregulated genes, respectively. Grey values represent the numbers of genes shared between the two comparisons.

to molecular function and six to cellular components (Supplementary Table B.3). Acyclic graphs resulting from the enrichment analysis and showing the top five and top five-related biological processes, molecular functions, and cellular components most affected at each time interval during early fruit set are provided in Supplementary Fig. A.5. Among biological processes, terms with reference to secondary metabolite biosynthesis and metabolism, cell wall (CW) component biosynthesis and organisation, carbohydrate and lipid biosynthesis, and metabolism were the most enriched at FS2 and FS3, followed by terms related to growth, morphogenesis, pollen germination, and the reproductive process. GO terms related to response to stress, abscission, and dehiscence were enriched only at FS3 when compared to FS1. Regarding molecular functions, terms were mostly related to acyl transferase and secondary metabolite synthase, oxidoreductase, lyase, and esterase activities. Regarding cellular components, the most enriched category was cell membrane. Among this category, pollen tube and mitochondrial membrane terms were found to be more present in FS2 and FS3 samples, respectively.

\subsection{Overall metabolomic profile}

From the 215 metabolites searched by the global metabolic analyses conducted, a total of 213 were detected in at least one of the conditions, and 40 changed in their abundance during the three fruit set stages (Supplementary Table B.4). The relative contents of 19 of the metabolites were altered between the first and second sampling points, and 28 were altered in the transition from FS1 to FS3 (11 and 17 were more and less abundant at FS3 compared to FS1, respectively) (Fig. 5).

PCoA showed that, based on $71.33 \%$ of the total variation explained by the combination of PC1 and PC2 endorsed by the metabolite profile, FS1 samples are different from FS2 and FS3, but these later two time points are indistinguishable based on their quantified metabolome (Fig. 6A). The observed dispersion between replicates of FS2 and FS3 in the plot can be explained only by biological variation. Individual-plot pairwise comparisons of FS1 with FS2 and FS1 with FS3 (Supplementary Fig. A.6) highlighted that FS1 samples are distinguishable from both FS2 and FS3 and that the transition from FS1 to FS3 would generate more clear information to understand metabolomic dynamics resulting from development. Hierarchical clustering (Fig. 6B) based on metabo- lite profile showed that only samples from FS1 formed a cluster that was significantly separated from the other samples. Regarding metabolite association, a cluster composed of putrescine, 2-isopropylmalate, tartarate, adenosine 5 '-monophosphate (AMP), galactinol, and adenosine, which showed to be increased in the transition from FS1 to FS2 and FS3, was separated from the other metabolites. Diaminopropane and $\gamma$-tocotrienol showed the opposite pattern and were grouped into a different cluster.

\subsection{Transcriptomic and metabolomic profile by functional category}

Fig. 7 shows the functional annotation distribution of the 269 DEGs, from which 64 and 205 were automatically and manually assigned to KOG categories, respectively. From the remaining 227 DEGs, 13 were classified as 'other function' and 214 as 'general and unknown function' (Supplementary Table B.2). Secondary metabolite biosynthesis, transport, and metabolism; carbohydrate transport and metabolism; and signal transduction mechanisms were the most representative functional categories of DEGs during both FS1-FS2 and FS2-FS3 time intervals. In the transition from FS1 to FS3, secondary metabolite biosynthesis, transport, and metabolism; carbohydrate transport and metabolism; and post-translational modification, protein turnover, and chaperones categories were enriched. Table 4 shows the summary of the DEGs during fruit set aggregated by gene family and functional category and numbers of genes up- and down-regulated between each time point.

Fig. 8A shows the list of 28 metabolites whose relative contents were significantly affected at FS3 with respect to FS1 and the metabolites' assigned functional categories, KEGG compound number, and respective fold change. Among these metabolites, the amino acid class was the most prevalent (28\%), followed by carbohydrate (25\%); secondary metabolism (14\%); lipid (11\%); cofactor, prosthetic group, and electron carrier (11\%); and nucleotide (11\%). The pattern of relative abundance of the eight most affected metabolites during flower-to-fruit transition is shown in Fig. 8B. Among these metabolites, 2-oxodipate, galactinol, and two metabolites from nucleotide metabolism (adenosine and AMP) were increased at FS3, while two metabolites from secondary metabolism (ferulate and gamma-tocotrienol), glycerol, and 2hydroxyplamitate decreased in abundance during the same period.

As shown in Fig. 9, when integrating enzymatic and metabolic changes on metabolic pathways, the flower-to-fruit transition stage shows it is composed of changes in specific steps mapped on diverse metabolic pathways. The complete enzyme identification among DEGs and respective fold changes are given in Supplementary Table B.3.

\subsubsection{Amino acid transport and metabolism}

Genes encoding adenylylsulphate reductases (EC 1.8.4.8) that participate in sulphur metabolism, a hypothetical protein involved in glutathione metabolism (EC 2.3.2.2), and amino acid and oligopeptide transporter-related genes were down-regulated. Genes encoding gelatinases and peroxidases (EC 1.11.1.7) involved in phenylalanine metabolism and encoding an endopeptidase were also differentially expressed. On the other hand, a global increase of some metabolites from amino acid metabolism was observed, composed of shikimate and tryptophan from the shikimate pathway; 2-oxodipate and 2-isopropylmalate, involved in lysine and leucine biosynthesis, respectively; and putrescine from polyamine metabolism. Aspartate accumulation showed the opposite pattern. An increase of methionine sulphoxide (the oxidised form of methionine) and a decrease of S-adenosylhomocysteine, with both metabolites putatively involved in ethylene biosynthesis, were verified. 
Table 4

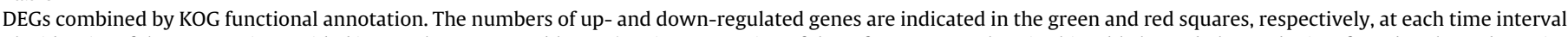

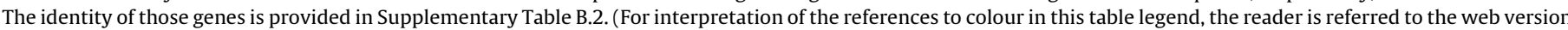
of this article.)

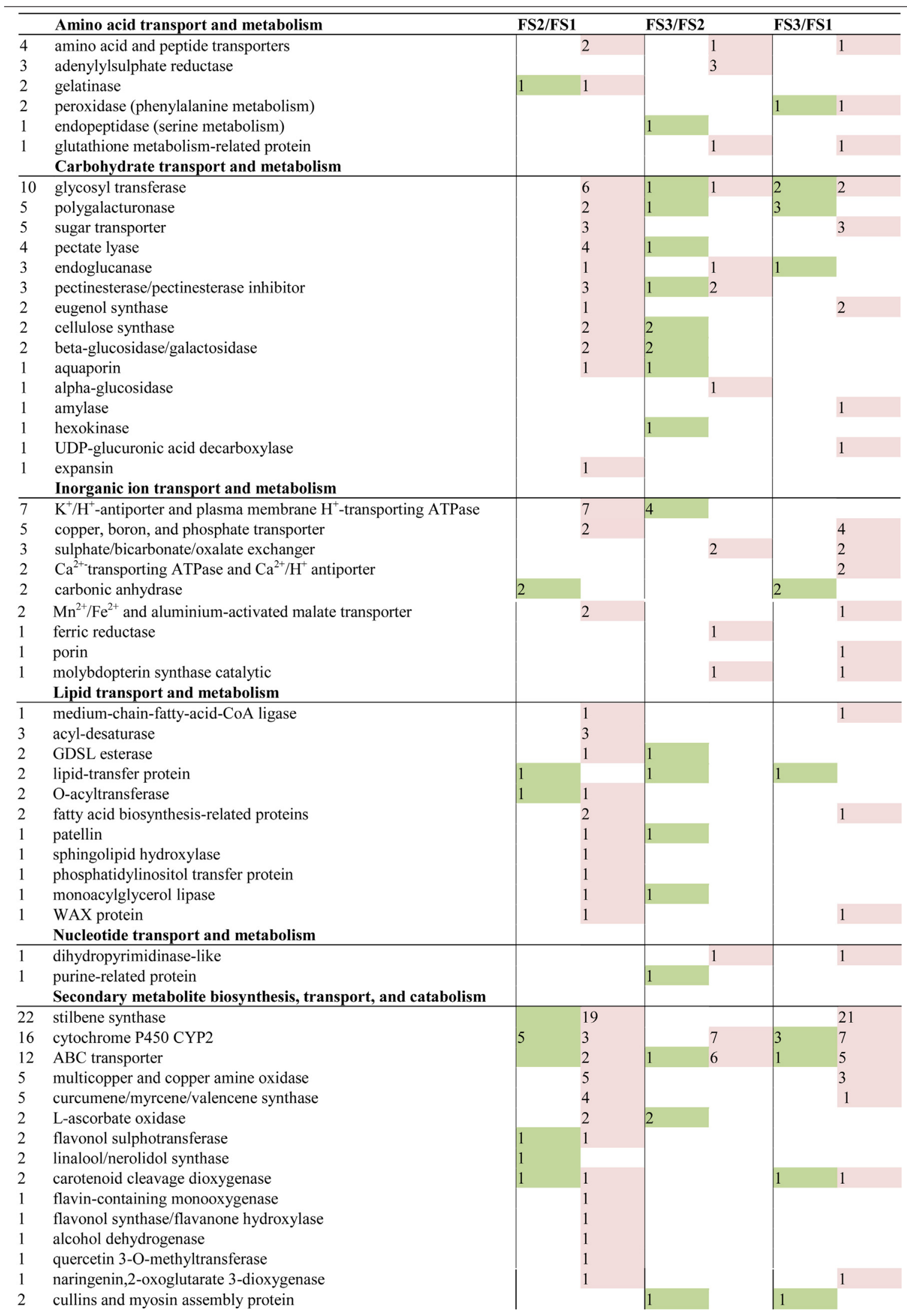


Table 4 (Continued)

Coenzyme transport and metabolism

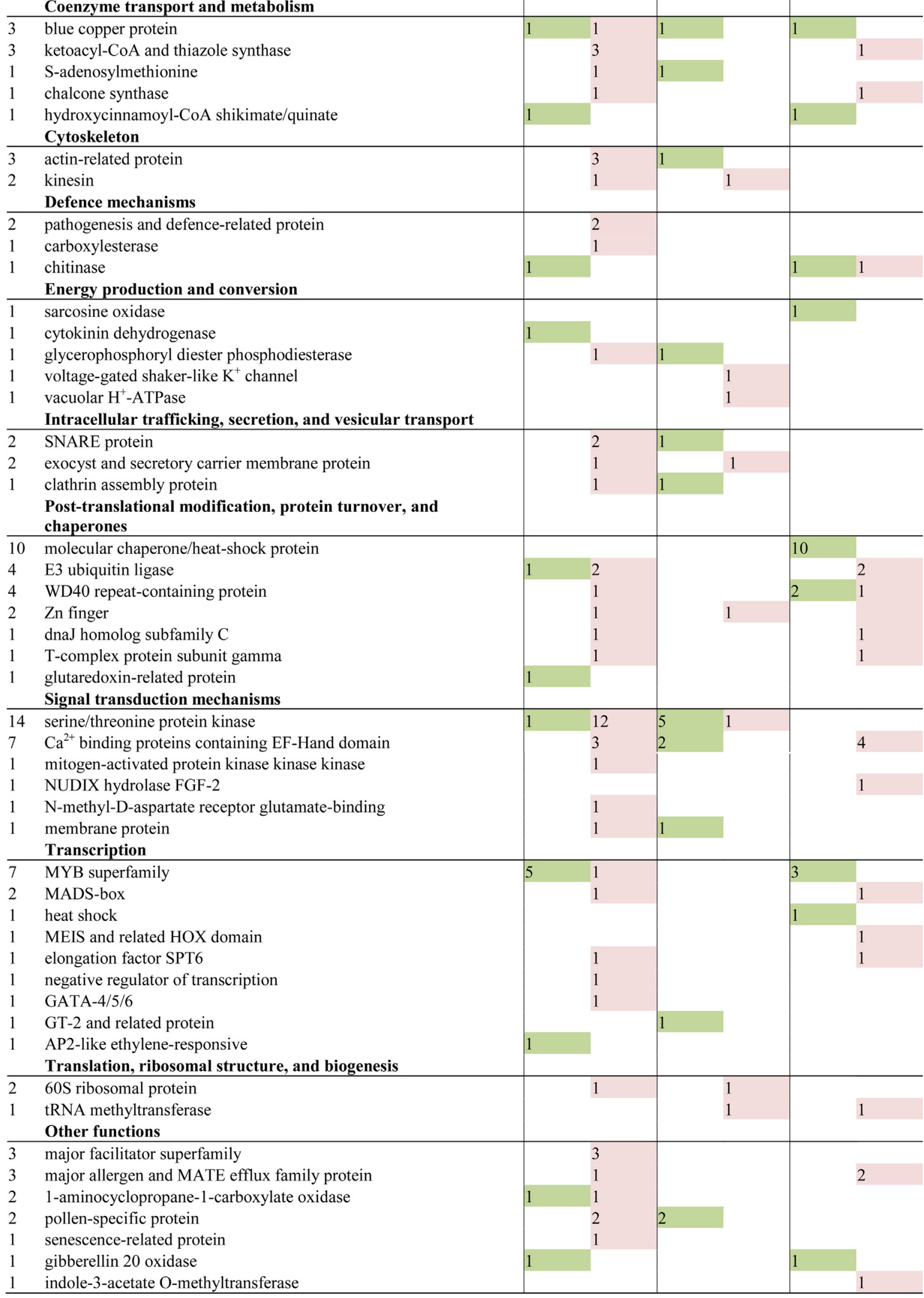


A

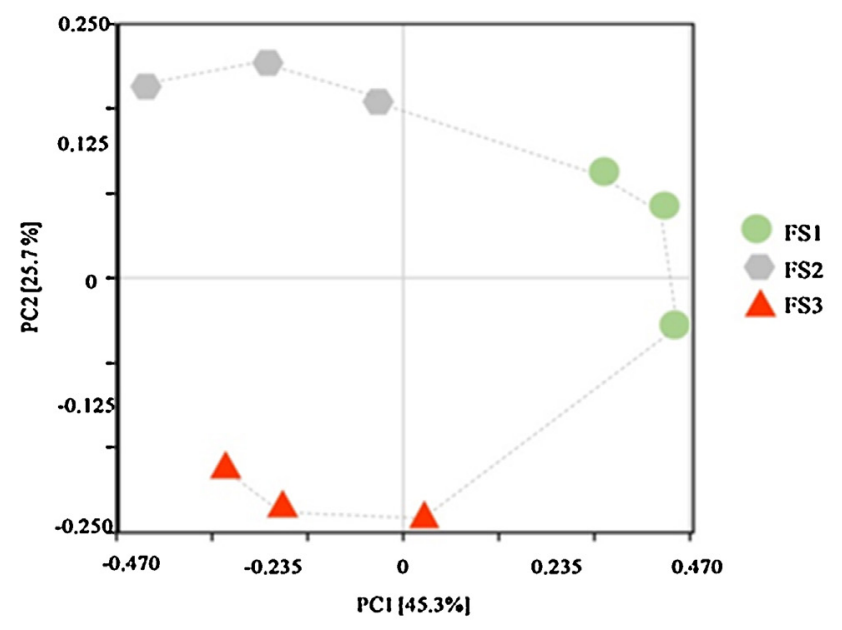

B
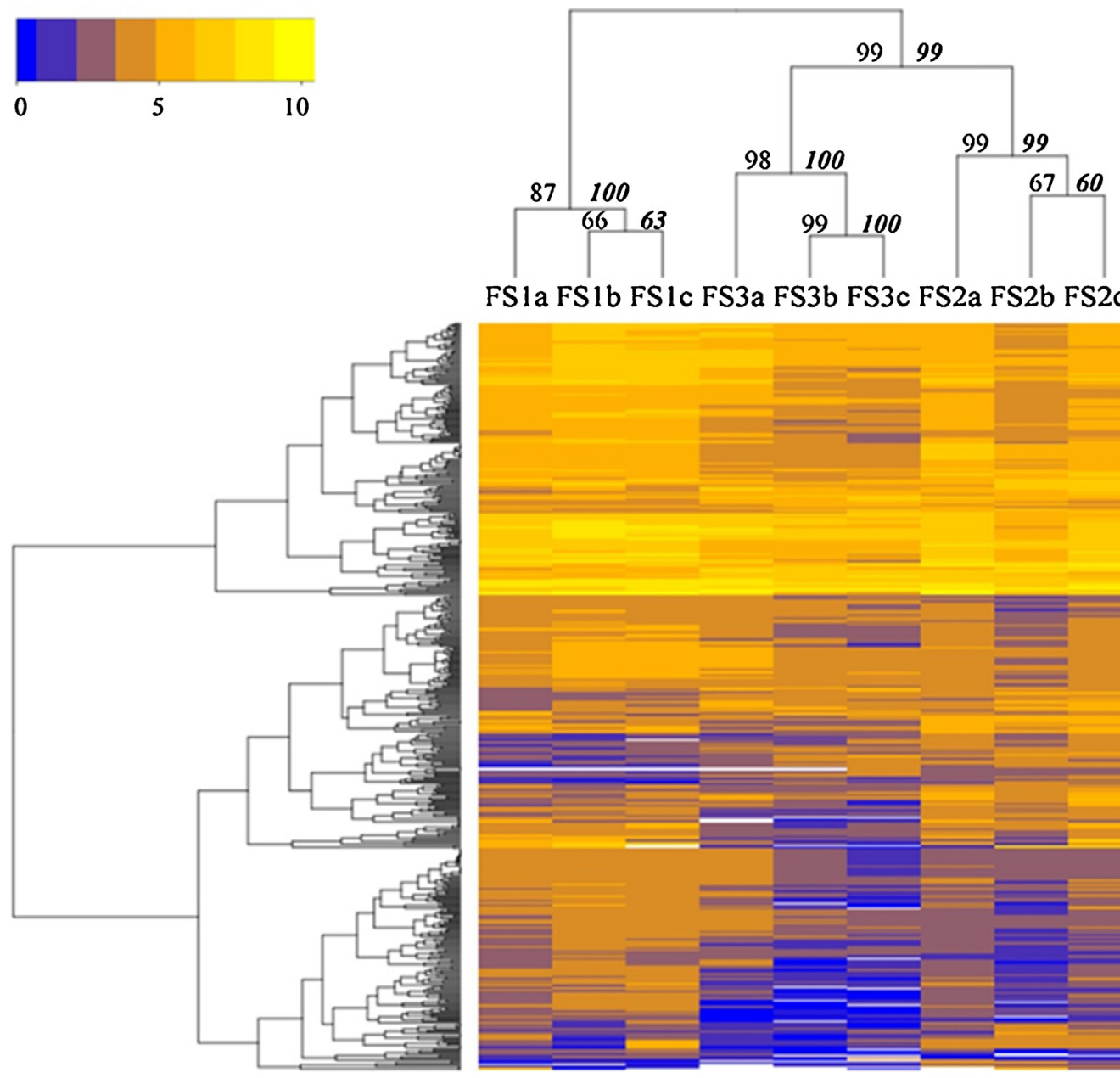

FS1a FS1b FS1c FS3a FS3b FS3c FS2a FS2b FS2c

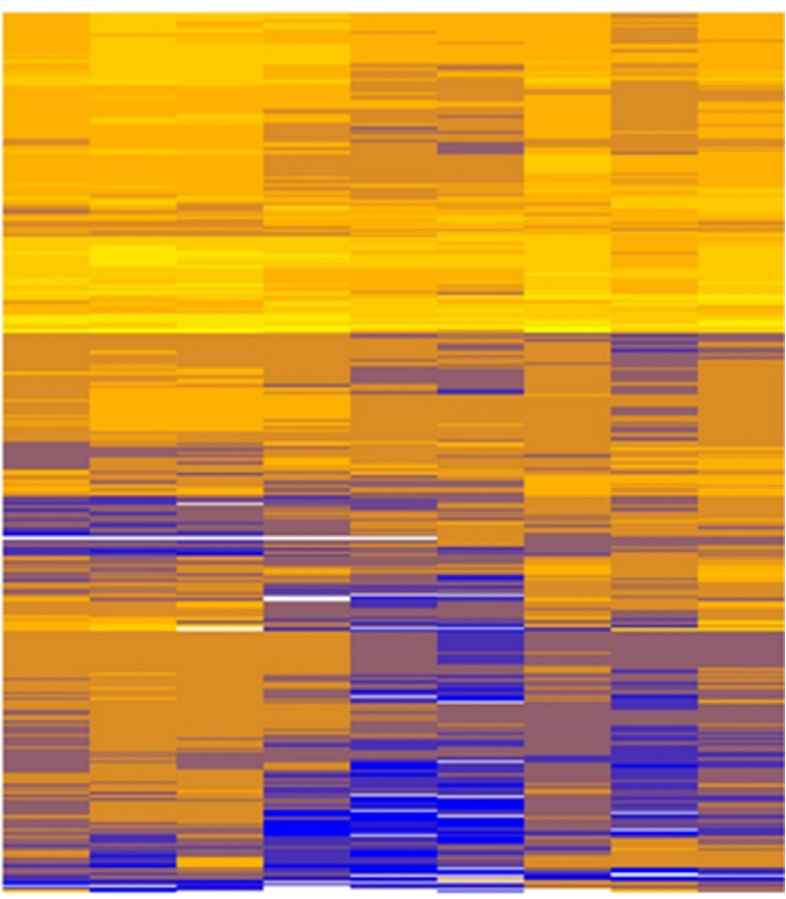

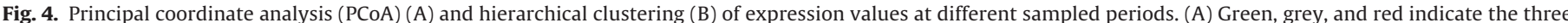

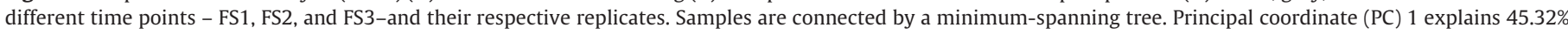

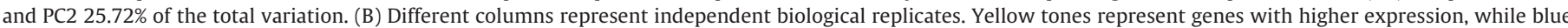

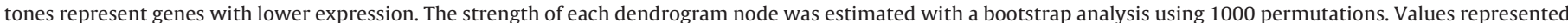

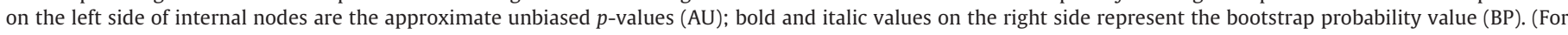
interpretation of the references to colour in this figure legend, the reader is referred to the web version of this article.)

\subsubsection{Carbohydrate transport and metabolism}

DEGs involved in carbohydrate metabolism were downregulated during the first time interval investigated, while, in the second interval, the majority of DEGs were up-regulated. Cluster 5 (Fig. 2) was highly represented within this functional category, including genes encoding cellulose synthases (EC 2.4.1.12), beta- 


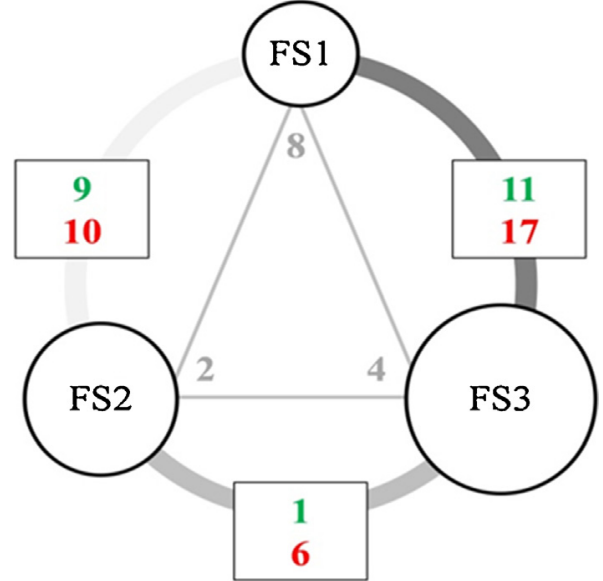

Fig. 5. Diagram showing the metabolites with significantly different abundances at each of the three time points sampled during early fruit set. Values indicate genes passing the $p$-value $\leq 0.05$ cutoff. Green and red values indicate numbers of metabolites with increased and decreased accumulation, respectively. Grey values represent the number of metabolites shared between the two comparisons.

galactosidases (EC 3.2.1.23), and a tonoplast-intrinsic aquaporin. However, genes encoding eugenol synthase, sugar transporters, expansin, amylase (EC 3.2.1.2), and UDP-glucuronic acid decarboxylase (EC 4.1.1.35) were exclusively down-regulated during the fruit set stages studied. Genes encoding proteins of glycosyl (EC 2.4.1.14), galactosyl, and galacturonosyl (E.C 2.4.1.134) transferases and $\mathrm{CW}$-related enzymes, such as members from pectate lyase (EC 4.2.2.2), endoglucanase, polygalacturonase (EC 3.2.1.15), glucosidase,and pectinesterase/pectinesterase inhibitor (EC 3.1.1.11) families, were also differentially expressed during the period investigated. Regarding metabolites, pyruvate, citrate, fumarate, and chiro-inositol were shown to be less abundant at FS3 than in FS1. Conversely, increased galactinol, ribitol, and tartarate were measured.

\subsubsection{Coenzyme transport and metabolism}

Genes encoding thiazole, ketoacyl-CoA, and chalcone synthase enzymes were down-regulated at FS2 compared to FS1, while hydroxycinnamoyl-CoA shikimate/quinate was up-regulated at the same period. S-adenosylmethionine (SAM) synthase (EC 2.5.1.6), involved in ethylene biosynthesis, and blue copper proteins were also differentially expressed. Nicotinate ribonucleoside and methylphosphate relative contents decreased during the stages investigated.

\subsubsection{Inorganic ion transport and metabolism and intracellular trafficking}

The majority of genes encoding inorganic ion transporter proteins showed a down-regulation pattern. Genes encoding $\mathrm{K}^{+} / \mathrm{H}^{+}$-antiporters and plasma membrane $\mathrm{H}^{+}$-transporting ATPase were down-regulated during the first time interval but shift to an up-regulation in the second interval, while carbonic anhydrases showed an up-regulated pattern during the whole period sampled. A down-regulation of members encoding exocyst components and secretory carrier membrane proteins was also perceived. Genes encoding SNARE and clathrin assembly proteins were downregulated at the first time interval and up-regulated at the second one.

\subsubsection{Lipid transport and metabolism}

The majority of genes involved in lipid transport and metabolism pathways were down-regulated at FS2 in comparison with FS1, including, among others, genes encoding medium-
chain-fatty-acid-CoA ligase (EC 6.2.1.25), acyl-desaturases (EC 1.14.19.2), and a WAX protein. Two of these genes, encoding patellin and monoacylglycerol lipase proteins, inverted the expression trend at FS3 compared to FS2. Non-specific lipid transfer proteins were up-regulated during both time intervals. Two genes that encode O-acyltransferases, O-ACYLTRANSFERASE WSD1 and $O$-ACYLTRANSFERASE WSD1-LIKE, assigned under the different enzymatic classifications EC 2.3.1.20 and EC 2.3.1.75, were downand up-regulated, respectively. Fatty acids, glycerolipids, and sterol metabolites showed decreased amounts in the sampled inflorescences during the stages investigated.

\subsubsection{Nucleotide transport and metabolism}

One gene classified to encode a dihydropyrimidinase (EC 3.5.2.2) was down-regulated, and other encoding a putative RNA polymerase (EC 2.7.7.6) was up-regulated during the transition from FS2 to FS3. Adenosine and AMP had accumulated at FS3 compared to FS1, while N6-carbamoylthreonyladenosine relative content decreased.

\subsubsection{Secondary metabolite biosynthesis, transport, and catabolism}

Most of the genes involved in secondary metabolite biosynthesis, transport, and catabolism showed a down-regulation pattern and cluster 2 (Fig. 2) was highly represented within this functional category, including 22 genes encoding stilbene synthases (EC 2.3.1.95), which are involved in phenylpropanoid metabolism, and myrcene (EC 4.2.3.15) and limonene synthases (EC 4.2.3.16, EC 4.2.3.20), known to be involved in terpenoid metabolism. On the other hand, genes encoding linalool and nerolidol synthases (EC 4.2.3.25), also involved in terpenoid metabolism, were up-regulated in the transition from FS1 to FS2. Genes encoding L-ascorbate oxidases, ABC transporters, cytochrome P450-related proteins, carotenoid cleavage dioxygenase, and flavonol sulphotransferase were among the other DEGs.

All metabolites assigned to the secondary metabolism functional category significantly decreased their abundance at FS3, namely gamma-tocotrienol from tocopherol metabolism, ferulate, involved in phenylpropanoid metabolism, procyanidin B1 and trimer from flavonoid metabolism, and hydroquinone betaD-glucopyranoside from the benzenoid family.

\subsubsection{Cell cycle control and cytoskeleton}

Genes from the cell cycle control, cell division, and chromosome partitioning category were up-regulated. Genes encoding actin-related proteins, assigned to cytoskeleton function, were down-regulated at FS2 compared to FS1 and up-regulated during the second time interval, while kinesin protein-related genes were down-regulated at both time intervals.

\subsubsection{Energy production and conversion}

Genes encoding sarcosine oxidase (EC 1.5.3.1) and cytokinin dehydrogenase (EC 1.5.99.12) were up-regulated, while those encoding a voltage-gated shaker and vacuolar $\mathrm{H}^{+}$-ATPase were down-regulated. One gene encoding a glycerophosphoryl diester phosphodiesterase was down-regulated at FS2 compared to FS1 but up-regulated during the last time interval.

\subsubsection{Transcription factors, translation, and post-translational modification}

Genes from the MYB transcription factor superfamily were mostly up-regulated during the timespan investigated, while MADS-box, MEIS and the related HOX domain, elongation factor SPT6, negative regulator of transcription and GATA-4/5/6 transcription factors were down-regulated. Genes encoding the 
A

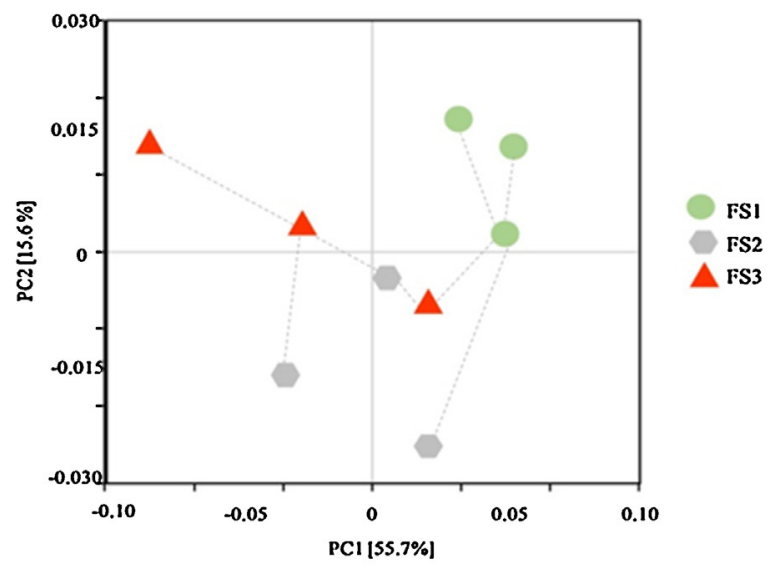

B
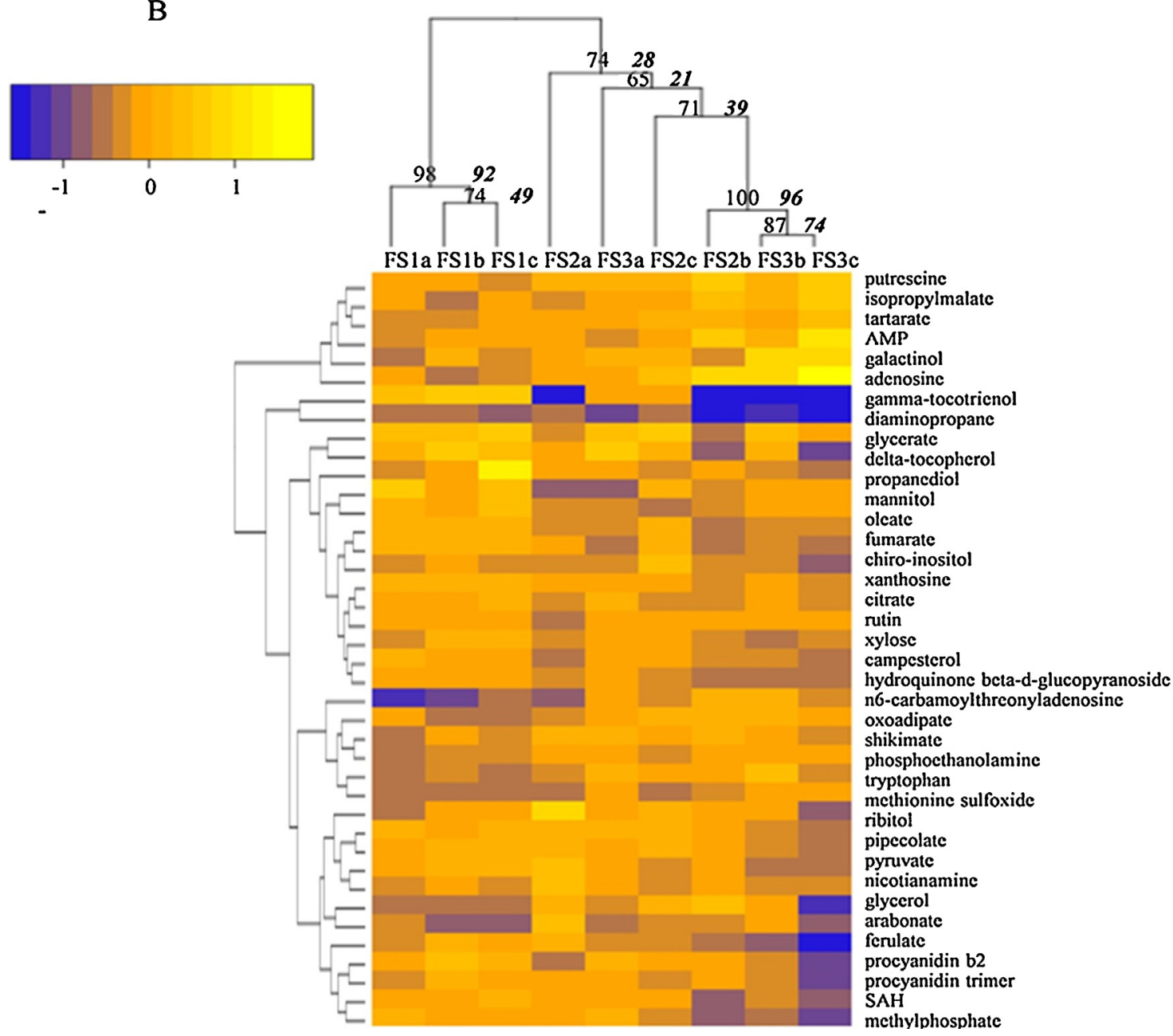

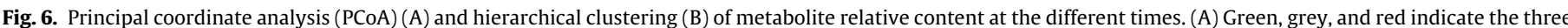

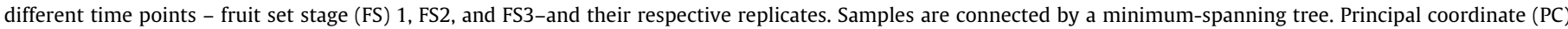

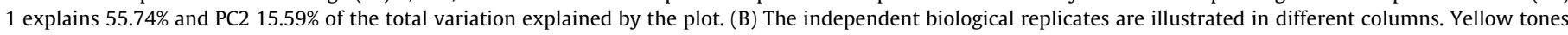

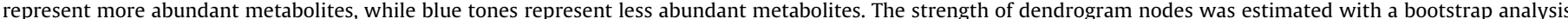

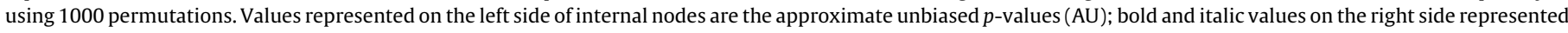
the bootstrap probability value (BP). (For interpretation of the references to colour in this figure legend, the reader is referred to the web version of this article.)

AP2-like ethylene-responsive factor, GT-2, and heat-shock transcription factors were up-regulated in the transition from FS1 to FS2, FS2 to FS3, and FS1 to FS3, respectively. Genes encoding a tRNA methyltransferase and ribosomal proteins, from the translation, ribosomal structure, and biogenesis functional category, were down-regulated in our sample set. Conversely, the major- ity of genes involved in post-translational modification, protein turnover, and chaperones showed an up-regulated pattern.

\subsubsection{Signal transduction, defence mechanisms, and other} functions

Regarding the signal transduction mechanisms, the majority of DEGs were down-regulated during the transition from FS1 to 
FS2/FS1

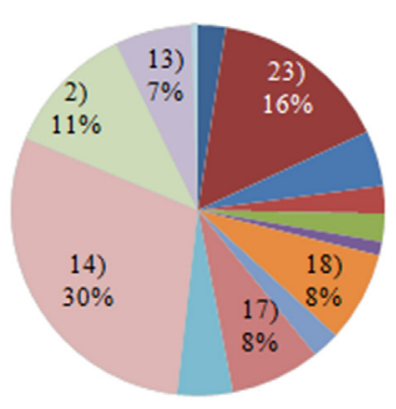

FS3/FS2

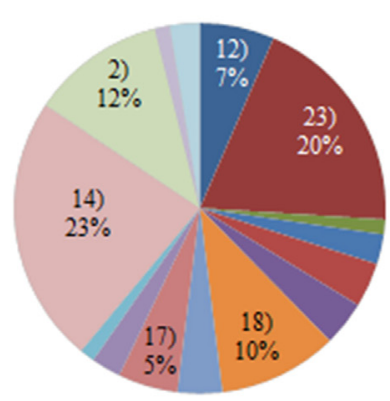

FS3/FS1

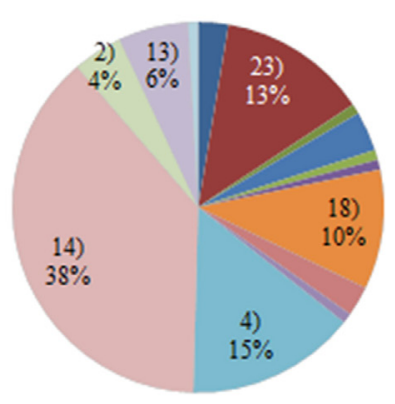

1) Translation and ribosomal structure 2) Signal transductionmechanisms 3) RNA processing andmodification 4) Posttranslationalmodification, protein tumover 5)Nuclear structure

6) Intracellular trafficking, secretion and vesicular transport

= 7) Extracellular structures

S) Defense mechanisms

- 9) Coenzyme transport and metabolism

- 10)Cell wall/membrane/envelope biogenesis

-11)Cell cycle control, division and chrom. part.

- 12)Amino acid transport andmetabolism
13) Transcription

14) Secondary metabolites biosynthesis, transport and metabolism

15) Replication, recombination and repair

16)Nucleotide transport andmetabolism

17) Lipid transport and metabolism

$=18$ ) Inorganic ion transport and metabolism

-19) Energy production and conversion

=20)Cytoskeleton

-21) Chromatin structure and dynamics

-22)Cell motility

- 23) Carbohydrate transport and metabolism

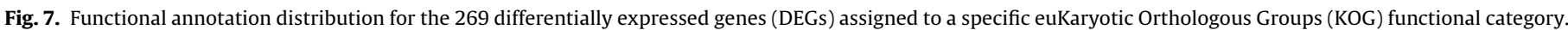

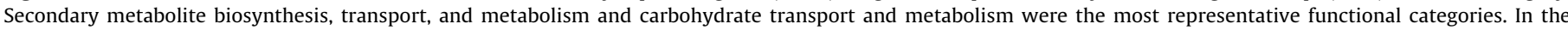
transition from FS1 to FS3, the post-translational modification, protein turnover and chaperones category also was particularly enriched.

FS2 and included genes encoding serine/threonine protein kinases, mitogen-activated protein kinase kinase kinases, and calciumbinding messenger proteins. Among these genes, five encoding serine/threonine proteins kinases, two calcium-binding messenger proteins, and one membrane protein were up-regulated from FS2 to FS3.

All DEGs identified involved in defence mechanisms were downregulated in the transition from FS1 to FS2 except the CHITINASE gene, which was up-regulated under a pattern represented by Cluster 8 (Fig. 2 and Table 4). Genes involved in hormone metabolism, namely 1-AMINOCYCLOPROPANE-1-CARBOXYLATE OXIDASE (ACO), GA20ox, and INDOLE-3-ACETATE-O-METHYLTRANSFERASE 1 (IAMT1) were differentially expressed. DEGs encoding a senescence-related protein, MATE efflux family proteins, major facilitator superfamily membrane transport proteins, and major allergen proteins were down-regulated during the time period targeted. Genes encoding a pollen-specific protein were down-regulated in the transition from FS1 to FS2 and up-regulated during the following time interval.

\section{Discussion}

\subsection{RNA-Seq computational data validation by analysis of variability of biological replicates}

Using next-generation sequencing technology as a convenient tool for comparative transcriptome analyses of species with or without genome sequences [54,55], the technical variability of the data is increasingly negligible, while the establishment of biological replicates allows for deciding whether observed differences between groups of organisms exposed to different treatments are simply random or represent a 'true' biological difference induced by a given treatment. Following the approach previously proposed $[56,57]$, the significant correlation verified between biological replicates used as part of the experimental design (Supplementary Fig. A.4) confirms that our RNA-Seq data is reproducible and precise. Previous studies addressing dichotomy between biological and technical replicates showed that biological variation is often larger than technical variation, underscoring the importance of including biological replicates in the study design [58]. Taking advantage of the use of biological replicates, the PCOA and hierarchical clustering confirmed the sample separation into three classes sharing similar expression signatures according to the specific time point during fruit set (Fig. 4).

\subsection{Gene expression and metabolic patterns during fruit set}

The general molecular dynamics that occurred during fruit set highlighted a gene down-regulation pattern observed for 5 days after $100 \%$ cap fall in most functional categories (Table 4) that was accompanied by decreased metabolite abundance, mainly concerning carbohydrates, cofactors, lipids, and secondary metabolism, during the last investigated time point (Fig. 8). In addition, a total of $12.5 \%$ of DEGs showed an inversion trend in expression patterns at FS2, indicating that at this time point a developmental or stress-resulting signal occurs. The same expression dynamics was observed in tomato for a short period, ranging from 2 days before bloom to 4 days after full bloom, demonstrating a lack of continuum in ovary development from flower bud to post-anthesis [24].

\subsection{Secondary metabolism regulation}

Functional annotation (Fig. 5) and the top five GO enriched terms (Supplementary Table B.2) highlighted secondary metabolism as the most representative biological process.

The large multigenic family of MYB transcription factors, with members known to be regulated by sugars and to control flavonoid 


\begin{tabular}{|c|c|c|}
\hline \multirow[b]{2}{*}{ Metabolite } & \multicolumn{2}{|l|}{$\log _{2}$} \\
\hline & FS3/FS1 & KEGG \\
\hline \multicolumn{3}{|l|}{ Amino acid } \\
\hline shikimate & 0.51 & C00493 \\
\hline tryptophan & 0.49 & C0007s \\
\hline 2-oxoadipate & 1.43 & $\operatorname{co0} 322$ \\
\hline aspartate & -0.64 & C00049 \\
\hline methionine sulfoxide & 0.96 & C029\$9 \\
\hline S-adenosylhomocysteine (SAH) & -0.62 & $\mathrm{C} 00021$ \\
\hline 2-isopropylmalate & 0.75 & $\mathrm{C} 02504$ \\
\hline putrescine & 0.75 & $\mathrm{C} 00134$ \\
\hline \multicolumn{3}{|l|}{ Carbohvdrate } \\
\hline pynuvate & -0.58 & $\mathrm{C} 00022$ \\
\hline citrate & -0.43 & C0015s \\
\hline fumarate & -0.74 & $\mathrm{C} 00122$ \\
\hline tartarate & 0.64 & coos9s \\
\hline ribitol & 0.75 & $\mathrm{C} 00474$ \\
\hline chiro-inositol & -0.94 & \\
\hline galactinol & 1.29 & $\mathrm{C} 01235$ \\
\hline \multicolumn{3}{|l|}{ Imids } \\
\hline 2-hydroxypalmitate & -1.84 & \\
\hline 1,2-propanediol & -1.29 & cooss3 \\
\hline campesterol & -0.56 & $\mathrm{C} 01789$ \\
\hline \multicolumn{3}{|c|}{ Cofactors. Prosthetic Groups. Electron Camiers } \\
\hline nicotinate ribonucleoside & -0.47 & $\cos \$ 41$ \\
\hline methylphosphate & -0.64 & \\
\hline \multicolumn{3}{|l|}{ Nucleotide } \\
\hline adenosine & 2.20 & $\mathrm{C} 00212$ \\
\hline adenosine 5'-monophosphate (AMP) & 1.20 & $\mathrm{C} 00020$ \\
\hline N6-carbamoylthre onyladenosine & -0.36 & \\
\hline \multicolumn{3}{|l|}{ Secondary metabolism } \\
\hline hydroquinone beta-D-glucopyranoside & -0.54 & C061S6 \\
\hline procyanidin B2 & -0.58 & C17639 \\
\hline ferulate & -1.18 & C01494 \\
\hline procyanidin trimer & -0.51 & \\
\hline gamma-tocotrienol & -2.00 & C14155 \\
\hline
\end{tabular}

B
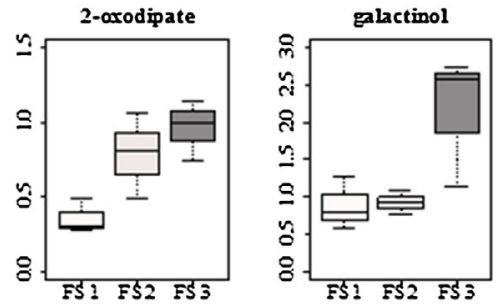

2-hyd roxip almitate
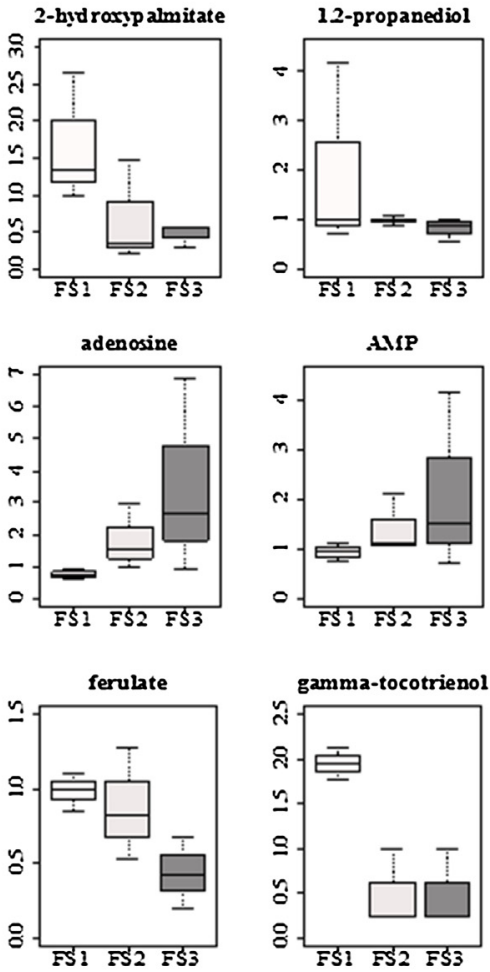

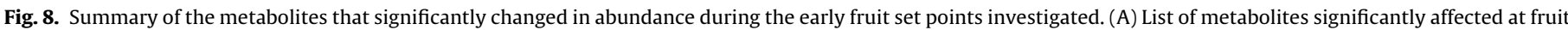

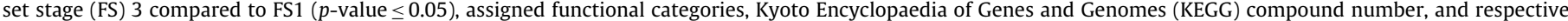
accumulation fold change. (B) Relative content evolution of all metabolites with highly significant differences (|fold change| $\geq 1$ ) between FS1 and FS3.

biosynthesis [59], showed up-regulation during fruit set (Table 4). Based on our results, we can hypothesise that the signalling sugar galactinol and hexokinase enzyme could be involved in regulation of MYB expression. Specific MYB transcription factors were previously described to be positive $[60,61]$ or negative [62] regulators of flavonoid biosynthesis in several species. Our data revealed repression of flavonoid- and phenylpropanoid-related pathways (Figs. 8 and 9) and repression of the phenylalanine precursor, $\beta$ alanine, biosynthesis via spermidine by primary amine oxidase (EC 1.4.3.21) and via uracil by dihydropyrimidinase (EC 3.5.2.2) (Fig. 9). The observed down-regulation of genes encoding $\mathrm{ABC}$ transporters (Table 4) indicated also reduced transport and accumulation of flavonoids in inflorescences during fruit set [63].

The regulation of cytochrome P450-related genes; terpenoid metabolism (EC 4.2.3.15, EC 4.2.3.25); protective compound biosynthesis, such as EUGENOL SYNTHASE 1-LIKE, LACCASE-14-LIKE, and CHITINASE; genes encoding ketoacyl-CoA synthases; and WAX2 protein, which contributes to cuticular wax and suberin biosynthesis, indicated that a response to environmental stimuli is activated during fruit set, agreeing with GO enriched terms associated with environmental stress responses and biotic stimuli (Table 4 and Supplementary Table B.3). The regulation of L-ASCORBATE OXIDASE HOMOLOG during the three fruit set stages investigated, with the change of expression pattern at FS2, also indicates a response to oxidative stress conditions.

\subsection{Regulation of nutrient transport in the developing fruit}

The passive symplastic phloem unloading pathway in the fruit wall and seed coat, through plasmodesmata, predominates during the early stage of berry development [64]. However, our data, which indicate that extensive transcriptome reprogramming of nutrient transporters (Table 4 ) is induced, agree with the evidence that nutrient transport across membranes requiring membraneembedded amino acids, peptides, sugar, secondary metabolites, and inorganic ion transporters is strongly regulated during fruit set $[25,65,66]$.

The observed down-regulation of membrane sugar transporter genes, namely those encoding sucrose transport protein SUC2 and bidirectional sugar transporter SWEET1, 4, 5, and 14, indicates a down-regulation of phloem long-distance transport (sucrose) and photosynthate short-term transport or accumulation (glucose) levels [67-70]. The expression of SWEET14 was found in the flowerto-fruit transition stage with a 4-fold repression at FS3 compared to FS1 (Supplementary Table B.2), suggesting a sink limitation for sugars previously observed in fruit set (reviewed by [25]). The 


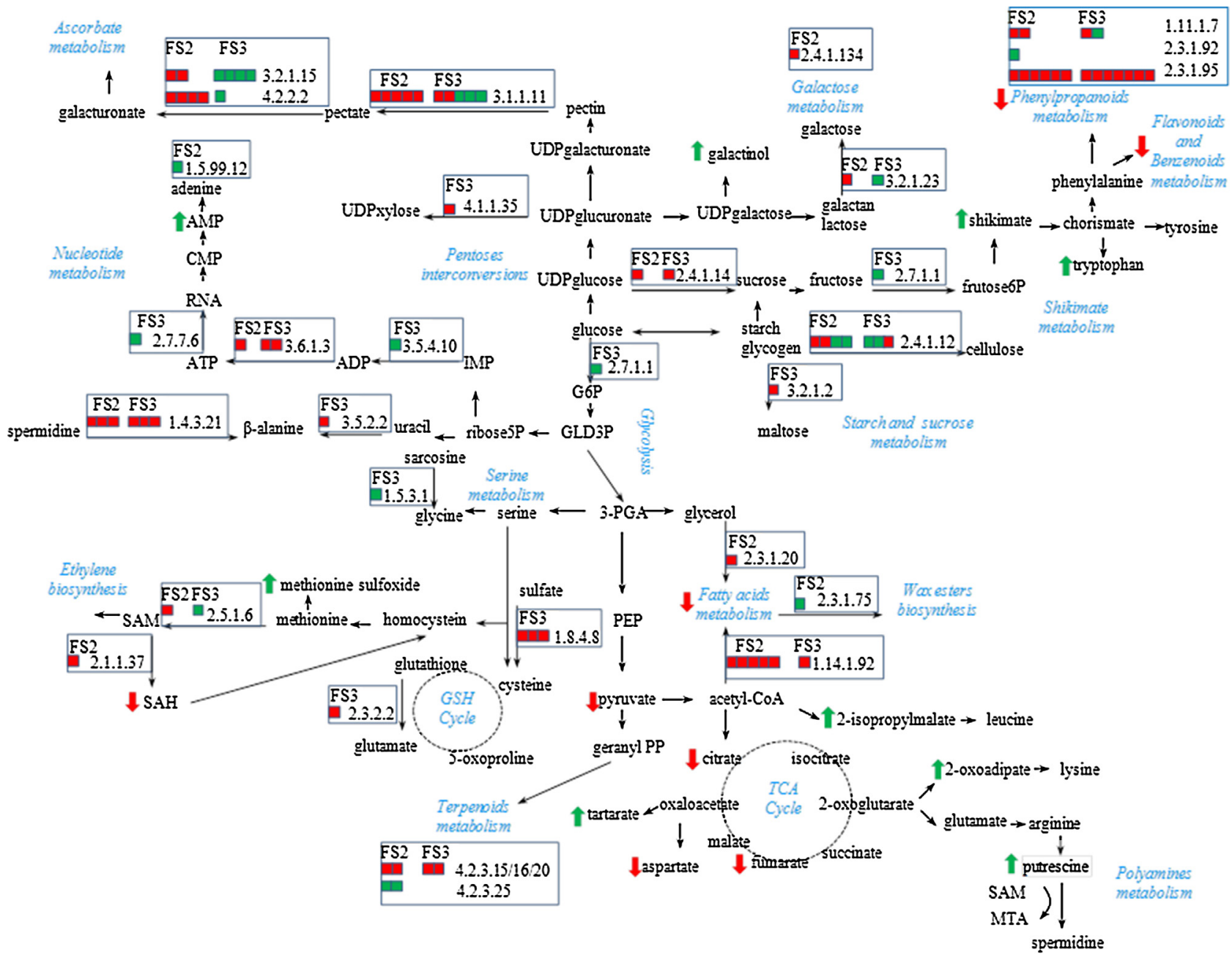

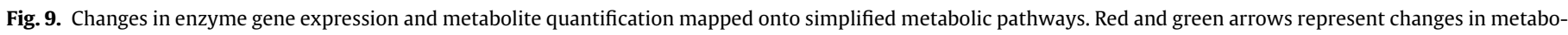

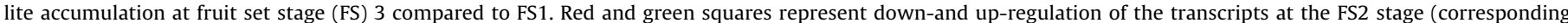

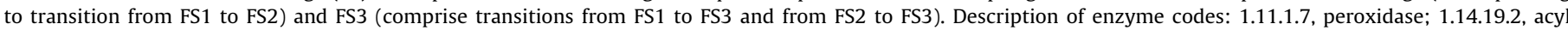

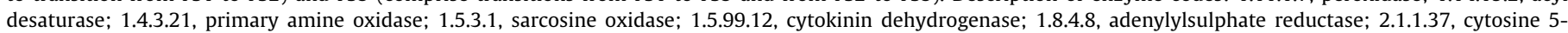

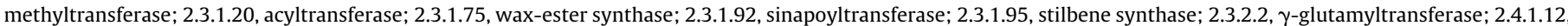

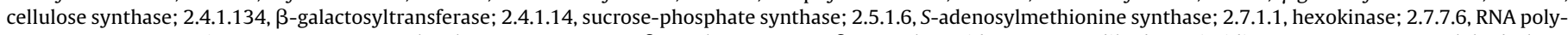

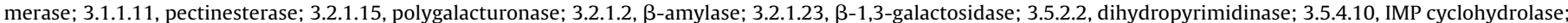

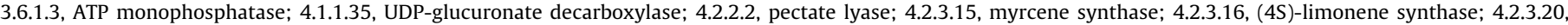

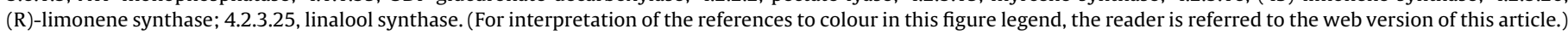

gene $V v S U C 2 / S U T 2$, which participates in phloem or post-phloem unloading, first described as weakly expressed in berries [71], was shown to be involved in onset of berry development. In addition to the known impaired flower formation and berry set resulting from carbon limitation due to inadequate rates of source leaf photosynthesis $[9,72]$, these results indicate the sink strength limitation is an important step during the flower-to-fruit transition stage.

\subsection{Carbohydrate metabolism and other energy sources}

The repression of carbohydrate-related pathways at the transcriptome level during the first time interval investigated, which can be correlated with decreased metabolite content associated with glycolysis and the tricarboxylic acid cycle, was inverted at FS2 (Fig. 8 and Table 4) and could indicate the starting point of cell metabolism stimulation that would lead to berry formation. The perceived up-regulation of two genes encoding glycogenin enzymes (VIT_07s0005g01970, VIT_07s0005g01980), among the glycosyltransferases, suggests that conversion of glucose to the energy storage polymer glycogen is favoured (Supplementary Table
B.2). The demand for energy can also be fulfilled by increased glycogen biosynthesis via sarcosine oxidase (EC 1.5.3.1). In addition, adenine synthesised via cytokinin dehydrogenase (EC 1.5.99.12) can be converted to adenosine with the increased levels observed and can be involved in energy transfer processes (ATP and ADP) (Figs. 8 and 9).

CW biosynthesis accompanies the mandatory remodelling that provides the flexibility required for cell expansion during fertilised flower and pedicel development $[22,24]$ and is fine-tuned to cope with the double sigmoidal growth pattern of berry development [73]. Evidence of CW remodelling processes was gained by changes in expression of genes encoding glycosyltransferase and cellulose synthase (EC 2.4.1.12) enzymes, which are required for pectin and cellulose biosynthesis; and expansins, endoglucanases, pectinesterases (EC 3.1.1.11), polygalacturonases (EC 3.2.1.15), and $\beta$-1,3-galactosidases (EC 3.2.1.23), which facilitate CW polymer modification (Fig. 9 and Table 4). Genes encoding CW disassemblyassociated enzymes can also be implicated in abscission of the non-fertilised flower organs [74], which are present in our whole inflorescence samples. This corroborates with the GO enrichment 
analysis (Supplementary Table B.3), which revealed terms related to $\mathrm{CW}$ organisation and modification and flower organ abscission biological processes.

\subsection{Sugar signalling pathways}

Sugars may control a wide range of activities in plant cells through transcriptional and post-translational regulation. The increased galactinol quantified during fruit set (Fig. 8) may affect expression of different genes, such as encoding sugar transporters, components of the photosystems, enzymes of carbohydrate metabolism, enzymes of secondary metabolism, and transcription factors [59]. The regulation of HEXOKINASE-1 (VIT_09s0002g03390) (Table 4) was reported to be involved in sugar-sensing and signalling through $\mathrm{CW}$ invertase and sucrose synthase regulation [75] and sugar control of transporters [59] in grape berries. Here, we can associate this regulation with early stages of fruit set in grapevine, during which hexose sugar signals that regulate the cell cycle and cell division programmes seemed to be generated [76]. The enhanced expression of genes encoding heat-shock proteins (HSP90, HSP70, and sHSP26/42) and one heat stress transcription factor (Table 4 and Supplementary Table B.2) can be hypothesised to protect $\mathrm{CW}$ invertase from misfolding for correct targeting and function [77]. These proteins are known to be expressed in response to stress conditions and during particular stages of the cell cycle [78], perform chaperone function by stabilising new proteins, and play important roles in signalling, trafficking, and pathogen resistance [79].

\subsection{Hormone metabolism and signalling pathways}

The discerned regulation of auxin-, GA-, and ethylene-related genes during fruit set (Table 4) confirms the importance of plant hormones as mediators of the fruit developmental signal after pollination [21-24]. Our data also revealed the mediation of auxin biosynthesis and signalling [80] via down-regulation of IAMT, an enzyme responsible for inactivation of indole-3-acetic acid (IAA), and down-regulation of auxin-associated MADS-box genes [24]. It can be speculated that these two MADS-box genes (VIT_14s0066g01640, VIT_18s0001g01760) (Supplementary Table B.2) are instrumental in triggering the grapevine fruit set programme. The up-regulation of GIBBERELLIN 20 OXIDASE 2 (GA20ox2) (Table 4) suggests that GA biosynthesis occurs during initial steps of fruit set, as previously proposed [21,81]. Additionally, the observed regulation of genes encoding predicted ACO (Table 4) and SAM synthase 1-like (EC 2.5.1.6) (Fig. 8) and the increased methionine sulphoxide content derived from the intermediate methionine indicated changes in ethylene-related pathways, as previously reported [24]. Likewise, the up-regulation of a gene encoding an AP2-like ethylene-responsive transcription factor suggested the activation of the ethylene signalling pathway to act as a fruit set trigger [24]. Since polyamines and ethylene share SAM as a common intermediate, SAM may be alternatively channelled toward the polyamine pathway, which is also closely related to fruit set [82]. The observed increase in putrescine indicated an induction of polyamine biosynthesis in the grapevine post-anthesis stage, which contrasts with the previous observation in tomato [24].

Therefore, it can be discussed that the down-regulation of this IAA inhibitor probably results in increased levels of active IAA, which, together with induction of ethylene signalling, would trigger fruit set and would stimulate the synthesis of bioactive GAs responsible for fruit growth through prompting cell division and expansion. On the other hand, the observed up-regulation of a cytokinin dehydrogenase (VIT_11s0016g02110) might be associated with a decrease in cytokinin levels. Previous work in grapevine demonstrated that an induction of cytokinin biosynthesis occurs about 13 days after pollination [83].

\subsection{Pollen viability, fertilisation, and cell division phase}

In 'Thompson Seedless', fruit set is dependent on successful pollination and fertilisation; however, seed development aborts at an early stage after fertilisation [12]. The observed regulation of genes related to the pollen viability (Table 4), including genes encoding enzymes responsible for cellulose synthesis (EC 2.4.1.12), pectate lyase (EC 4.2.2.2), amylase (EC 3.2.1.2), and acyl-CoA synthase, identified specific members for synthesis of energy, cellulose, callose, and sporopollenin for pollen development [84-86]. Pollen tube CW development also requires changes in lipid metabolism, such as palmitate that can be converted into 2-hydroxypalmitate, which showed decreased relative abundance 3-7d (Fig. 8). Changes in gene expression involved in active vesicle trafficking to deliver secretory vesicles suggest polarised rapid growth pollen tube regulation via exo- and endocytosis [87] (Table 4). This evidence and the regulation of genes encoding pollen-specific proteins from the major latex protein regulatory family [88] agrees with the enriched GO terms corresponding to anther dehiscence biological processes and pollen tube cellular components.

Simultaneously, during fruit set stages investigated, a period of rapid cell division takes place, as verified by up-regulation of genes assigned to the cell cycle control, cell division, and chromosome partitioning functional category (Table 4), corresponding to ovary development [3].

\section{Conclusions}

During the decisive fruit set developmental stage, carbohydrate pathway, secondary metabolism, and protein modification and turnover mechanisms were the most affected functional categories. An increased cell division-related gene expression, required for fruit growth initiation, was verified. Genes encoding signalling proteins, such as serine/threonine kinases and calciumbinding messenger proteins, L-ascorbate oxidase, chitinase, and CW-modifying enzymes, showed a discontinuous participation in this developmental stage with a change in expression pattern at FS2. It was also shown to be characterised by pathways that mediate sugar- and hormone-controlled responses. The participation of auxin, GA, ethylene, and cytokinin-related genes from the earlier fruit set stage investigated (FS1), maintaining the same expression pattern until FS3, suggests that hormone action is one of the constant trigger signals needed for the flower-to-fruit transition to proceed. Therefore, we propose that repression of nutrient transport together with increased levels of active IAA and induction in ethylene signalling will trigger fruit set and stimulate the synthesis of bioactive GAs responsible for fruit growth through stimulation of cell division and expansion. Therefore, this first global transcriptomic and metabolomic analyses help elucidate the molecular mechanisms occurring in inflorescences during the flower-to-fruit transition stage.

\section{Acknowledgements}

This work was supported by the Portuguese Fundação para a Ciência e a Tecnologia (FCT) through project 'VitiShade: PTDC/AGRGPL/116923/2010', the Ph.D. grant SFRH/BD/69076/2010 to SD, and by ProDeR Action4.1: PROdUVA 23921/2/3/4. We acknowledge Dr. Danny Alexander and his team at Metabolon (Durham, USA) for metabolomic analyses and Dr. Martin Heine and his team at LGC Genomics (Berlin, Germany) for transcriptome sequencing. 


\section{Appendix A. Supplementary data}

Supplementary data associated with this article can be found, in the online version, at http://dx.doi.org/10.1016/j.plantsci.2015.12. 009.

\section{References}

[1] B.G. Coombe, Relationship of growth and development to changes in sugars, auxins, and gibberellins in fruit of seeded and seedless varieties of Vitis vinifera, Plant Physiol. 35 (1960) 241-250.

[2] N.K. Dokoozlian, Grape berry growth and development, in: L.P. Christensen (Ed.), Raisin Production Manual, University of California, Agricultural and Natural Resources, Oakland, 2000, pp. 30-37.

[3] H. Ojeda, A. Deloire, A. Carbonneau, A. Ageorges, C. Romieu, Berry development of grapevines: relations between the growth of berries and their DNA content indicate cell multiplication and enlargement, Vitis 38 (1999) $145-150$.

[4] C. Pratt, Reproductive anatomy in cultivated grapes: a review, Am. J. Enol. Vitic. 22 (1971) 92-109.

[5] J.S. Boyer, J.E. McLaughlin, Functional reversion to identify controlling genes in multigenic responses: analysis of floral abortion, J. Exp. Bot. 58 (2007) 267-277.

[6] Y. Jin, D.A. Ni, Y.L. Ruan, Post-translational elevation of cell wall invertase activity by silencing its inhibitor in tomato delays leaf senescence and increases seed weight and fruit hexose level, Plant Cell 21 (2009) 2072-2089.

[7] W.M. Kliewer, Effect of high temperatures during the bloom-set period on fruit-set ovule fertility, and berry growth of several grape cultivars, Am. J. Enol. Vitic. 28 (1977) 215-222.

[8] H. Suwa, H. Hakata, H.A. El-Shemy, N.T. Adu-Gyamfi, S. Nguyen, P.K. Lightfoot, K. Mohapatra, High temperature effects on photosynthate partitioning and sugar metabolism during ear expansion in maize (Zea mays L.) genotypes, Plant Physiol. Biochem. 48 (2010) 124-130.

[9] M.C. Vasconcelos, M. Greven, C.S. Winefield, M.C.T. Trought, V. Raw, The flowering process of Vitis vinifera: a review, Am. J. Enol. Vitic. 60 (2009) 411-434.

[10] K.E. Zinn, M. Tunc-Ozdemir, J.J. Harper, Temperature stress and plant sexual reproduction: uncovering the weakest links, J. Exp. Bot. 61 (2010) 1959-1968.

[11] C.C. Nwafor, I. Gribaudo, A. Schneider, R. Wehrens, M.S. Grando, L. Costantini, Transcriptome analysis during berry development provides insights into co-regulated and altered gene expression between a seeded wine grape variety and its seedless somatic variant, BMC Genomics 15 (2014) 1030-1051.

[12] F. Varoquaux, R. Blanvillain, M. Delseny, P. Gallois, Less is better: new approaches for seedless fruit production, Trends Biotechnol. 18 (2000) 233-242.

[13] G. Lebon, B. Wojnarowiez, F. Holzapfel, N. Vaillant-Gaveau, C. Clément, Sugars and flowering in the grapevine (Vitis vinifera L.), J. Exp. Bot. 59 (2008) 2565-2578.

[14] D. Werner, N. Gerlitz, R. Stadler, A dual switch in phloem unloading during ovule development in Arabidopsis, Protoplasma 248 (2011) 225-235.

[15] C. Zhang, K. Tanabe, F. Tamura, K. Matsumoto, A. Yoshida, ${ }^{13} \mathrm{C}$-photosynthate accumulation in Japanese pear fruit during the period of rapid fruit growth is limited by the sink strength of fruit rather than by the transport capacity of the pedicel, J. Exp. Bot. 56 (2005) 2713-2719.

[16] M. De Jong, M. Wolters-Arts, R. Feron, C. Mariani, W.H. Vriezen, The Solanum lycopersicum auxin response factor 7 (SlARF7) regulates auxin signaling during tomato fruit set and development, Plant J. 57 (2009) 160-170.

[17] M. De Jong, M. Wolters-Arts, C. García-Martínez, W.H. Vriezen, The Solanum lycopersicum auxin response factor 7 (SlARF7) mediates cross-talk between auxin and gibberellin signalling during tomato fruit set and development, J Exp. Bot. 62 (2011) 617-626.

[18] I. Olimpieri, F. Siligato, R. Caccia, L. Mariotti, N. Ceccarelli, G.P. Soressi, A. Mazzucato, Tomato fruit set driven by pollination or by the parthenocarpic fruit allele are mediated by transcriptionally regulated gibberellin biosynthesis, Planta 226 (2007) 877-888.

[19] L. Pascual, J.M. Blanca, J. Cañizares, F. Nuez, Analysis of gene expression during the fruit set of tomato: a comparative approach, Plant Sci. 173 (2007) 609-620.

[20] Z. Ren, Z. Li, Q. Miao, Y. Yang, W. Deng, Y. Hao, The auxin receptor homologue in Solanum lycopersicum stimulates tomato fruit set and leaf morphogenesis, J. Exp. Bot. 62 (2011) 2815-2826.

[21] J.C. Serrani, R. Sanjuán, O. Ruiz-Rivero, M. Fos, J.L. García-Martínez, Gibberellin regulation of fruit set and growth in tomato, Plant Physiol. 145 (2007) 246-257.

[22] W.H. Vriezen, R. Feron, F. Maretto, J. Keijman, C. Mariani, Changes in tomato ovary transcriptome demonstrate complex hormonal regulation of fruit set, New Phytol. 177 (2008) 60-76.

[23] H. Wang, B. Jones, Z. Li, P. Frasse, C. Delalande, F. Regad, S. Chaabouni, A. Latché, J.C. Pech, M. Bouzayen, The tomato Aux/IAA transcription factor IAA9 is involved in fruit development and leaf morphogenesis, Plant Cell 17 (2005) 2676-2692.

[24] H. Wang, N. Schauer, B. Usadel, P. Frasse, M. Zouine, M. Hernould, A. Latché, J.C. Pecha, A.R. Fernie, M. Bouzayen, Regulatory features underlying pollination-dependent and -independent tomato fruit set revealed by transcript and primary metabolite profiling, Plant Cell 21 (2009) 1428-1452.

[25] Y.L. Ruan, J.W. Patrick, M. Bouzayen, S. Osorio, A.R. Fernie, Molecular regulation of seed and fruit set, Trends Plant Sci. 17 (2012) 656-665.

[26] J.E. McLaughlin, J.S. Boyer, Sugar-responsive gene expression, invertase activity, and senescence in aborting maize ovaries at low water potentials, Ann. Bot. 94 (2004) 675-689.

[27] P. De Dios, A.J. Matilla, M. Gallardo, Flower fertilization and fruit development prompt changes in free polyamines and ethylene in damson plum (Prunus insititia L.), J. Plant Physiol. 163 (2006) 86-97.

[28] O. Jaillon, J.M. Aury, B. Noel, A. Policriti, C. Clepet, A. Casagrande, N. Choisne, S Aubourg, N. Vitulo, C. Jubin, et al., The grapevine genome sequence suggests ancestral hexaploidization in major angiosperm phyla, Nature 449 (2007) 463-467.

[29] R. Velasco, A. Zharkikh, M. Troggio, D.A. Cartwright, A. Cestaro, D. Pruss, M. Pindo, L.M. FitzGerald, S. Vezzulli, J. Reid, et al., A high quality draft consensus sequence of the genome of a heterozygous grapevine variety, PLoS One 2 (2007) 1326-1343.

[30] R. Cramer, K.A. Ghan, R.L. Schlauch, H. Tillett, A. Heymann, M. Ferrarini, S. Delledonne, M. Zenoni, Transcriptomic analysis of the late stages of grapevine (Vitis vinifera cv. Cabernet Sauvignon) berry ripening reveals significant induction of ethylene signaling and flavor pathways in the skin, BMC Plant Biol. 14 (2014) 370-390.

[31] L.G. Deluc, J. Grimplet, M.D. Wheatley, R.L. Tillett, D.R. Quilici, C. Osborne, D.A. Schooley, K.A. Schlauch, J.C. Cushman, G.R. Cramer, Transcriptomic and metabolite analyses of Cabernet Sauvignon grape berry development, BMC Genomics 8 (2007) 429-470.

[32] J. Grimplet, L.G. Deluc, R.L. Tillett, M.D. Wheatley, K.A. Schlauch, G.R. Cramer, J.C. Cushman, Tissue-specific mRNA expression profiling in grape berry tissues, BMC Genomics 8 (2007) 187-209.

[33] C. Sweetman, D.C. Wong, C.M. Ford, D.P. Drew, Transcriptome analysis at four developmental stages of grape berry (Vitis vinifera cv. Shiraz) provides insights into regulated and coordinated gene expression, BMC Genomics 13 (2012) 691-715.

[34] L. Venturini, A. Ferrarini, S. Zenoni, G.B. Tornielli, M. Fasoli, S. Dal Santo, A. Minio, G. Buson, P. Tononi, E.D. Zago, G. Zamperin, D. Bellin, M. Pezzotti, M. Delledonne, De novo transcriptome characterization of Vitis vinifera cv. Corvina unveils varietal diversity, BMC Genomics 14 (2013) 41-53.

[35] S. Zenoni, A. Ferrarini, E. Giacomelli, L. Xumerle, M. Fasoli, G. Malerba, D. Bellin, M. Pezzotti, M. Delledonne, Characterization of transcriptional complexity during berry development in Vitis vinifera using RNA-Seq, Plant Physiol. 152 (2010) 1787-1795.

[36] D. Lorenz, K. Eichhorn, H. Bleiholder, R. Klose, U. Meier, E. Weber, Phänologische entwicklungsstadien der rebe (Vitis vinifera L. ssp. vinifera): codierung und beschreibung nach der erweiterten BBCH-Skala, Vitic. Enol. Sci. 49 (1994) 66-70.

[37] S. Chang, J. Cairney, A simple and efficient method for isolating RNA from pine trees, Plant Mol. Biol. Rep. 11 (1993) 113-116

[38] A.M. Bolger, M. Lohse, B. Usadel, Trimmomatic: a flexible trimmer for Illumina sequence data, Bioinformatics 30 (2014) 2114-2120.

[39] D. Kim, G. Pertea, C. Trapnell, H. Pimentel, R. Kelley, S.L. Salzberg, TopHat2: accurate alignment of transcriptomes in the presence of insertions, deletions, and gene fusions, Genome Biol 14 (2013) 36-48.

[40] C. Trapnell, B.A. Williams, G. Pertea, A. Mortazavi, G. Kwan, M.J. van Baren, S.L. Salzberg, B.J. Wold, L. Pachter, Transcript assembly and quantification by RNA-Seq reveals unannotated transcripts and isoform switching during cell differentiation, Nat. Biotechnol. 28 (2010) 511-515.

[41] L. Kumar, M.E. Futschik, Mfuzz: a software package for soft clustering of microarray data, Bioinformation 2 (2007) 5-7.

[42] Y. Shen, Z. Wan, C. Coarfa, R. Drabek, L. Chen, E.A. Ostrowski, Y. Liu, G.M. Weinstock, D.A. Wheeler, R.A. Gibbs, F. Yu, A SNP discovery method to assess variant allele probability from next-generation resequencing data, Genome Res. 20 (2010) 273-280.

[43] M.I. Love, W. Huber, S. Anders, Moderated estimation of fold change and dispersion for RNA-Seq data with DESeq2, Genome Biol. 15 (2014) 550-570.

[44] Y. Zhao, H. Tang, Y. Ye, RAPSearch2: a fast and memory-efficient protein similarity search tool for next-generation sequencing data, Bioinformatics 28 (2012) 125-126

[45] R.L. Tatusov, N.D. Fedorova, J.D. Jackson, A.R. Jacobs, B. Kiryutin, E.V. Koonin, D.M. Krylov, R. Mazumder, S.L. Mekhedov, A.N. Nikolskaya, B.S. Rao, S. Smirnov, A.V. Sverdlov, S. Vasudevan, Y.I. Wolf, J.J. Yin, D.A. Natale, The COC database: an updated version includes eukaryotes, BMC Bioinformatics 4 (2003) 41-54.

[46] M. Kanehisa, M. Araki, S. Goto, M. Hattori, M. Hirakawa, M. Itoh, T. Katayama, S. Kawashima, S. Okuda, T. Tokimatsu, Y. Yamanishi, KEGG for linking genomes to life and the environment, Nucleic Acids Res. 36 (2008) 480-484.

[47] A. Conesa, S. Götz, J.M. García-Gómez, J. Terol, M. Talón, M. Robles, Blast2GO a universal tool for annotation, visualization, and analysis in functional genomics research, Bioinformatics 21 (2005) 3674-3676.

[48] A. Alexa, J. Rahnenfuhrer, topGO: enrichment analysis for gene ontology. R Package version 2.18.0, 2010

[49] A. Evans, C. DeHaven, T. Barrett, Integrated, nontargeted ultrahigh performance liquid chromatography/electrospray ionization tandem mass spectrometry platform for the identification and relative quantification of the small-molecule complement of biological systems, Anal. Chem. 81 (2009) 6656-6667. 
[50] A.M. Evans, M.W. Mitchell, H. Dai, C.D. DeHaven, Categorizing ion: features in liquid chromatography/mass spectrometry metobolomics data, Metabolomics 2 (2012) 110.

[51] T. Ohta, N. Masutomi, N. Tsutsui, T. Sakairi, M. Mitchell, M.V. Milburn, J.A. Ryals, K.D. Beebe, L. Guo, Untargeted metabolomic profiling as an evaluative tool of fenofibrate-induced toxicology in Fischer 344 male rats, Toxicol. Pathol. 37 (2009) 521-535.

[52] F.J. Rohlf, NTsys-PC: Numerical Taxonomy and Multivariate Analysis System, Exeter Software, Setauket, New York, 2005.

[53] R. Suzuki, H. Shimodaira, Pvclust: an R package for assessing the uncertainty in hierarchical clustering, Bioinformatics 22 (2006) 1540-1542.

[54] L. Gao, Z.J. Tu, B.P. Millett, J.M. Bradeen, Insights into organ-specific pathogen defense responses in plants: RNA-Seq analysis of potato tuber-Phytophthora infestans interactions, BMC Genomics 14 (2013) 340-351.

[55] J.J. Liu, R.N. Sturrock, R. Benton, Transcriptome analysis of Pinus monticola primary needles by RNA-Seq provides novel insight into host resistance to Cronartium ribicola, BMC Genomics 14 (2013) 884-899.

[56] M. Fasoli, S. Dal Santo, S. Zenoni, G.B. Tornielli, L. Farina, A. Zamboni, A. Porceddu, L. Venturini, M. Bicego, V. Murino, A. Ferrarini, M. Delledonne, M. Pezzotti, The grapevine expression atlas reveals a deep transcriptome shift driving the entire plant into a maturation program, Plant Cell 24 (2012) 3489-3505.

[57] D. Thakare, R. Yang, J.G. Steffen, J. Zhan, D. Wang, R.M. Clark, X. Wang, R. Clark, $\mathrm{X}$. Xiangfeng, R. Yadegari, RNA-Seq analysis of laser-capture microdissected cells of the developing central starchy endosperm of maize, Genomics Data 2 (2014) 242-245.

[58] J.H. Bullard, E. Purdom, K.D. Hansen, D. Dudoit, Evaluation of statistica methods for normalization and differential expression in mRNA-Seq experiments, BMC Bioinformatics 11 (2010) 94-106.

[59] F. Lecourieux, C. Kappel, D. Lecourieux, A. Serrano, E. Torres, P. Arce-Johnson, S. Delrot, An update on sugar transport and signalling in grapevine, J. Exp. Bot. 65 (2013) 821-832.

[60] J. Bogs, F.W. Jaffé, A.M. Takos, A.R. Walker, S.P. Robinson, The grapevine transcription factor VvMYBPA1 regulates proanthocyanidin synthesis during fruit development, Plant Physiol. 143 (2007) 1347-1361.

[61] A. Gonzalez, M. Zhao, J.M. Leavitt, A.M. Lloyd, Regulation of the anthocyanin biosynthetic pathway by the TTG1/bHLH/Myb transcriptional complex in Arabidopsis seedlings, Plant J. 53 (2008) 814-827.

[62] J.S. Park, J.B. Kim, K.J. Cho, C.I. Cheon, M.K. Sung, M.G. Choung, K.H. Roh, Arabidopsis R2R3-MYB transcription factor AtMYB60 functions as a transcriptional repressor of anthocyanin biosynthesis in lettuce (Lactuca sativa), Plant Cell Rep. 27 (2008) 985-994.

[63] E. Braidot, M. Zancani, E. Petrussa, C. Peresson, A. Bertolini, S. Patui, F. Macrì, A. Vianello, Transport and accumulation of flavonoids in grapevine (Vitis vinifera L.), Plant Signal. Behav. 3 (2008) 626-632.

[64] X.Y. Zhang, X.L. Wang, X.F. Wang, G.H. Xia, Q.H. Pan, R.C. Fan, F.O Wu, X.C. Yu, D.P. Zhang, A shift of phloem unloading from symplasmic to apoplasmic pathway is involved in developmental onset of ripening in grape berry, Plant Physiol. 142 (2006) 220-232.

[65] A. Sanders, R. Collier, A. Trethewy, G. Gould, R. Sieker, M. Tegeder, AAP1 regulates import of amino acids into developing Arabidopsis embryos, Plant J. 59 (2009) 540-552.

[66] M.C. Shelden, S.M. Howitt, B.N. Kaiser, S.D. Tyerman, Identification and functional characterisation of aquaporins in the grapevine, Vitis vinifera, Funct. Plant Biol. 36 (2009) 1065-1078.

[67] L.O. Chen, B.H. Hou, S. Lalonde, H. Takanaga, M.L. Hartung, X.Q. Qu, W.J. Guo, J.J. Kim, W. Underwood, B. Chaudhuri, D. Chermak, G. Antony, F.F. White, S.C. Somerville, M.B. Mudgett, W.B. Frommer, Sugar transporters for intercellular exchange and nutrition of pathogens, Nature 468 (2010) 527-532.

[68] J. Chong M.C. Piron, S. Meyer, D. Merdinoglu, C. Bertsch, P. Mestre, The SWEET family of sugar transporters in grapevine: VvSWEET4 is involved in the interaction with Botrytis cinerea, J. Exp. Bot. 65 (2014) 6589-6601.

[69] K. Shiratake, Genetics of sucrose transporter in plants, Genes Genom. Genomics 1 (2007) 74-80.
[70] T.L. Slewinski, Diverse functional roles of monosaccharide transporters and their homologs in vascular plants: a physiological perspective, Mol. Plant 4 (2011) 641-662

[71] D. Afoufa-Bastien, A. Medici, J. Jeauffre, P. Coutos-Thévenot, R. Lemoine, R. Atanassova, M. Laloi, The Vitis vinifera sugar transporter gene family: phylogenetic overview and macroarray expression profiling, BMC Plant Biol. 10 (2010) 245-266.

[72] S. Domingos, P. Scafidi, V. Cardoso, A.E. Leitao, R. Di Lorenzo, C.M. Oliveira, L.F. Goulao, Flower abscission in Vitis vinifera L. triggered by gibberellic acid and shade discloses differences in the underlying metabolic pathways, Front. Plant Sci. 6 (2015) 457-474.

[73] J.C. Goulão, P. Fernandes, Tackling the cell wall of the grape berry, in: M.M. Gerós (Ed.), The Biochemistry of the Grape Berry, Bentham Science Publishers, Sharjah, 2012, pp. 172-193.

[74] C. Ruperti, F. Bonghi, S. Ziliotto, A. Pagni, S. Rasori, P. Varotto, J.J. Tonutti, Characterization of a major latex protein (MLP) gene down-regulated by ethylene during peach fruitlet abscission, Plant Sci. 163 (2002) 265-272.

[75] X.Q. Wang, L.M. Li, P.P. Yang, C.L. Gong, The role of hexokinases from grape berries (Vitis vinifera L.) in regulating the expression of cell wall invertase and sucrose synthase genes, Plant Cell Rep. 33 (2014) 337-347.

[76] S. Bihmidine, C.T. Hunter, C.E. Johns, K.E. Koch, D.M. Braun, Regulation of assimilate import into sink organs: update on molecular drivers of sink strength, Front. Plant Sci. 4 (2013) 177-191.

[77] Z. Li, W.P. Palmer, A.P. Martin, R. Wang, F. Rainsford, Y. Jin, J.W. Patrick, Y. Yang, Y.L. Ruan, High invertase activity in tomato reproductive organs correlates with enhanced sucrose import into, and heat tolerance of, young fruit, J. Exp. Bot. 63 (2012) 1155-1166.

[78] E. Vierling, The roles of heat-shock proteins in plants, Annu. Rev Plant Physiol. Plant Mol. Biol. 42 (1991) 579-620.

[79] M.H. Al-Whaibi, Plant heat-shock proteins: a mini review, J. King Saud Univ. Sci. 23 (2011) 139-150.

[80] J. Li, R. Yuan, NAA and ethylene regulate expression of genes related to ethylene biosynthesis, perception, and cell wall degradation during fruit abscission and ripening in 'Delicious' apples, J. Plant Growth Regul. 27 (2008) 283-295.

[81] L. Giacomelli, O. Rota-Stabelli, D. Masuero, A.K. Acheampong, M. Moretto, L. Caputi, U. Vrhovsek, C. Moser, Gibberellin metabolism in Vitis vinifera L. during bloom and fruit-set: functional characterization and evolution of grapevine gibberellin oxidases, J. Exp. Bot. 64 (2013) 4403-4419.

[82] A. Aziz, Spermidine and related metabolic inhibitors modulate sugar and amino acid levels in Vitis vinifera L.: possible relationships with initial fruitlet abscission, J. Exp. Bot. 54 (2003) 355-363.

[83] P. Dauelsberg, J.T. Matus, M.J. Poupin, A. Leiva-Ampuero, F. Godoy, A. Vega, P. Arce-Johnson, Effect of pollination and fertilization on the expression of genes related to floral transition, hormone synthesis, and berry development in grapevine, J. Plant Physiol. 168 (2011) 1667-1674.

[84] C. De Azevedo Souza, S.S. Kim, S. Koch, L. Kienow, K. Schneider, S.M. McKim, G.W. Haughn, E. Kombrink, C.J. Douglas, A novel fatty acyl-CoA synthetase is required for pollen development and sporopollenin biosynthesis in Arabidopsis, Plant Cell 21 (2009) 507-525.

[85] A. Krichevsky, S.V. Kozlovsky, G.W. Tian, M.H. Chen, A. Zaltsman, V. Citovsky, How pollen tubes grow, Dev. Biol. 303 (2007) 405-420.

[86] S.G. Palusa, M. Golovkin, S.B. Shin, D.N. Richardson, A.S.N. Reddy, Organ-specific, developmental, hormonal, and stress regulation of expression of putative pectate lyase genes in Arabidopsis, New Phytol. 174 (2007) 537-550.

[87] L. Zonia, T. Munnik, Uncovering hidden treasures in pollen tube growth mechanics, Trends Plant Sci. 14 (2009) 318-327.

[88] B.L. Lytle, J. Song, N.B. de la Cruz, F.C. Peterson, K.A. Johnson, C.A. Bingman, G.N. Phillips, B.F. Volkman, Structures of two Arabidopsis thaliana major latex proteins represent novel helix-grip folds, Proteins 76 (2009) 237-243. 